\title{
Why is Consumption More Log Normal Than Income? Gibrat's Law Revisited
}

\author{
Erich Battistin \\ University of Padova and Institute for Fiscal Studies \\ Richard Blundell \\ University College London and Institute for Fiscal Studies \\ Arthur Lewbel \\ Boston College \\ Original: October 1999 \\ Revised: June 2007
}

\begin{abstract}
Significant departures from log normality are observed in income data, in violation of Gibrat's law. We identify a new empirical regularity, which is that the distribution of consumption expenditures across households is, within cohorts, closer to log normal than the distribution of income. We explain these empirical results by showing that the logic of Gibrat's law applies not to total income, but to permanent income and to maginal utility. These findings have important implications for welfare and inequality measurement, aggregation, and econometric model analysis.
\end{abstract}

Key Words: Consumption, Income, Lognormal, Inequality, Gibrat.

JEL Classification: D3, D12, D91

Acknowledgments: Funding for this research was provided by the ESRC centre for the analysis of Public Policy at the IFS. Data from the FES made available from the CSO through the ESRC data archive has been used by permission of the Controller of HMSO. We are responsible for all errors and interpretations. 


\section{Introduction}

A long-standing economic puzzle is the question of why the income distribution has a shape that is close to, but not quite, log normal. This is illustrated by Figure 1, which shows characteristics of the distribution of logged real income for a sample of households in the US consumer expenditure surveys from 1980 to 1989 (details regarding these figures are provided in section 4). The traditional parameterization of the income distribution is log normal with a thick, Pareto upper tail. The classic explanation for log normality of income is Gibrat's (1931) law, which essentially models income as an accumulation of random multiplicative shocks, however, the observed systematic departures from log normality have not been satisfactorily explained.

In this paper we first identify some new empirical regularities. One finding is that the distribution of consumption is also close to log normal, and is in fact closer to log normal than income. This can be seen in Figure 2, which shows the distribution of logged real consumption expenditures on nondurables and services for a birth-cohort of US households. This log normality of consumption within groups is not just a feature of US data, for example, Figure 3, shows the distribution of consumption for a demographically homogeneous group of United Kingdom households.

We now have two puzzles: why are both consumption and income approximately log normal, and why, within cohorts, is consumption much closer to log normal than income? We show that standard models of consumption and income evolution can explain both puzzles. In particular, the usual decomposition of an individual's income evolution process into permanent and transitory components is shown to imply that Gibrat's law applies to permanent income rather than total income. Similarly, standard Euler equation models make Gibrat's law apply to marginal utility and hence to consumption. The result is that the consumption distribution is closer to log normal than the income distribution within cohorts, and observed departures from log normality in the income distribution are attributable to non lognormality of the distribution of transitory income shocks across households.

These findings have important implications for welfare and inequality measurement, aggregation, and econometric model analysis, and may give rise to regularities in the distributions of other variables. Some examples of these implications are as follows: 
1. Log normal distributions result in simple expressions for aggregate models involving consumption or permanent income. See, e.g., Aitchison and Brown (1957), Doorn (1975), and Lewbel $(1990,1992)$.

2. Log normality implies that within cohorts, any measure of inequality, such as a Gini coefficient or the Lorenz curve, can be expressed as a function of a single scalar, the variance of log consumption (or equivalently, the coefficient of variation of consumption itself). This in turn implies that social welfare functions can be parsimoniously specified.

3. Banks, Blundell, and Lewbel (1997) exploit log normality of total consumption to simplify the handling of possible measurement errors in a nonlinear demand model.

4. Gabaix (1999) shows that Zipf's (1949) law for city populations may arise from an application of Gibrat's law to individual cities in a steady state. Analogous regularities may arise in consumption from Gibrat's law.

5. The budget shares for some goods, such as food, are known to be close to log linear in total consumption (see, e.g., Lewbel 1991 for US data and Banks, Blundell, and Lewbel 1997 for UK data), and hence can be expected to have a normal distribution across consumers.

In the next section we show why the logic behind Gibrat's law applies to permanent income rather than total income. In Section 3 we show how standard Euler equation models of consumption also yield Gibrat's law. The remainder of the paper is then devoted to detailed empirical analyses of the distributions of income and consumption by cohort, based on multiple surveys of United States and United Kingdom data.

\section{The Income Process and Log Normality}

For an individual that has been earning an income for $\tau$ years, let $y_{\tau}$ and $y_{\tau}^{p}$ be the individual's log income and log permanent income, respectively, so

$$
y_{\tau}=y_{\tau}^{p}+u_{\tau}
$$

where $u_{\tau}$ is defined as the transitory shock in log income. Permanent income evolves as

$$
y_{\tau}^{p}=y_{\tau-1}^{p}+\eta_{\tau}
$$

where $\eta_{\tau}$ is the shock to permanent income and $\eta_{1}$ is permanent income in the initial time period. In the above definitions it is assumed that the annuitized contributions of transitory 
income to future permanent income have been removed from $u_{\tau}$ and included in $\eta_{\tau}$. For example, all shocks to income in the final year of a person's life would be permanent shocks. This formalization of Friedman's (1957) decomposition of current income into permanent and transitory components is a common model of income behavior, see, e.g., MaCurdy (1982) and Meghir and Pistaferri (2004). This permanent income model implies that

$$
\frac{y_{\tau}^{p}}{\tau}=\frac{\sum_{s=1}^{\tau} \eta_{s}}{\tau}
$$

where $\tau$ is the number of time periods that the person has been earning an income, or more formally the number of periods for the income process.

Since $y_{\tau}^{p} / \tau$ is a simple average of random shocks, by application of a central limit theorem (CLT) assuming standard regularity conditions (e.g., shocks $\eta_{\tau}$ that satisfy a mixing process and have moments higher than two) there exists moments $\mu_{p}$ and $\sigma_{p}^{2}$ such that

$$
y_{\tau}^{p} \approx N\left(\tau \mu_{p}, \sigma_{p}^{2}\right)
$$

for large $\tau$. Therefore, the standard income generation model implies that permanent income (scaled by age $\tau$ ) should be close to log normally distributed, at least for individuals that are old enough to have experienced a moderate number of permanent income shocks. In particular, if permanent income were observable, the model would imply that the distribution of permanent income across individuals in the same (working) age cohort should be close to $\log$ normal.

The CLT also immediately implies Deaton and Paxson's (1994) result that the dispersion of income within cohorts increases with the age of the cohort. This follows from $E\left(y_{\tau}^{2}\right)=$ $\left[E\left(y_{\tau}^{p}+u_{\tau}\right)^{2}\right]=\tau^{2} \mu_{p}^{2}+\sigma_{p}^{2}+E\left(u_{\tau}^{2}\right)$, which grows with $\tau$. Our derivation here shows that not only does the standard model make dispersion of log income increase with age as Deaton and Paxson (1994) observe, but that the distribution becomes more normal as well. In fact, the observation that Gibrat's law implies a growing second moment was noted as early as Kalecki (1945).

Gibrat's original law assumed that income is determined by the accumulation of a series of proportional shocks. We have shown here that the standard permanent income model implies that it is permanent income, not total income, that is determined by an accumulation of 
shocks, and therefore that Gibrat's law should hold for permanent income, but not necessarily for total income.

If the transitory shocks $u_{\tau}$ are small relative to $y_{\tau}^{p}$ then log total income will also be approximately normal, but unless transitory shocks are themselves normally distributed, log permanent income will be closer to normal than total income. In particular, if transitory shocks have an appropriately skewed distribution (perhaps through some combination of overtime and temporary layoffs, or occasional large wealth shocks such as bequest receipts) then the total income distribution can take the classic empirical form of log normal with a Pareto upper tail.

\section{Euler Equations and Log Normality of Consumption}

An individual's permanent income is not directly observable. In this section we show that intertemporal utility maximization implies a similar structure for consumption, resulting from the cumulation of random shocks to income and other variables that affect utility. Traditional models of consumer behavior going at least as far back as Friedman (1957) assume that consumption is at least approximately equal to permanent income, and so the results of the previous section directly imply normality of log consumption in traditional models. In this section we obtain a similar result directly from consumption Euler equations.

Let $c_{\tau}$ be an individual's $\log$ real consumption at age $\tau$, and let $x_{\tau}$ be a vector of income and other variables that affect utility. These other variables could include lagged $c$ 's to permit habit effects, as well as prices, wages, demographic characteristics, stocks of durables, etc.,.

Assume that in each time period $\tau$ the individual maximizes the expectation of the time separable utility function

$$
u\left(c_{\tau}, x_{\tau}\right)+\sum_{s=\tau+1}^{T} \delta_{\tau+1} \ldots \delta_{s} u\left(c_{s}, x_{s}\right)
$$

subject to the expectation of the intertemporal budget constraint

$$
c_{\tau}-y_{\tau}+\sum_{s=\tau+1}^{T} R_{\tau} \ldots R_{s}\left(c_{s}-y_{s}\right)=w_{\tau}
$$

where $\delta_{\tau}$ is the individual's age $\tau$ subjective discount rate, $R_{\tau}$ is the market discount rate when the individual is aged $\tau$, and $w_{\tau}$ is accumulated wealth at age $\tau$, which can include 
a desired bequest, appropriately time discounted. Budget constrained maximization of this utility function yields the standard Euler equation model for consumption, (see, e.g., Deaton 1994 and references therein, beginning with Hall 1978), which is

$$
\phi\left(c_{\tau}, x_{\tau}\right)=b_{\tau} \phi\left(c_{\tau-1}, x_{\tau-1}\right)+e_{\tau}^{*}
$$

Here $\phi_{\tau}=\phi\left(c_{\tau}, x_{\tau}\right)=\partial u\left(c_{\tau}, x_{\tau}\right) / \partial c_{\tau}$ is the marginal utility of consumption, $e_{\tau}^{*}$ is the shock to consumption resulting from new information at age $\tau$, and $b_{\tau}=\delta_{\tau} / R_{\tau}$. Define $e_{1}^{*}=\phi_{1}$, and define $\varepsilon_{\tau \tau}=e_{\tau}^{*}$ and $\varepsilon_{\tau s}=b_{\tau} b_{\tau-1} \ldots b_{s+1} e_{s}^{*}$ for $s=0, \ldots, \tau-1$. Then

$$
\phi\left(c_{\tau}, x_{\tau}\right)=\sum_{s=1}^{\tau} \varepsilon_{\tau s} .
$$

Assuming the $\varepsilon_{\tau s}$ terms satisfy the conditions required for a triangular array CLT, there exists moments $\mu_{\phi}$ and $\sigma_{\phi}^{2}$ such that

$$
\phi\left(c_{\tau}, x_{\tau}\right) \approx N\left(\tau \mu_{\phi}, \sigma_{\phi}^{2}\right)
$$

for large $\tau$. There are many alternative regularity conditions that will yield a CLT here (see, e.g., Wooldridge and White 1988), but they all require a uniform asymptotic negligibility condition (relating to existence of moments) and a limit on the degree of dependence of observations over time such as alpha mixing. These conditions permit $\varepsilon_{\tau s}$ to be autocorrelated (by, e.g., the presence of $b_{\tau}$ terms, or because of taste changes over time), however, any such conditions will require that the $b_{\tau}$ terms be centered around one to avoid having $b_{\tau} b_{\tau-1} \ldots b_{s+1}$, and hence $\varepsilon_{\tau s}$, go to zero or infinity as $\tau$ goes to infinity.

This derivation shows that marginal utility $\phi$ should be close to normal, so if $\phi(c, x)$ is approximately linear in $c$, then logged consumption $c$ will also be close to log normal. Alternatively, expanding $\phi\left(c_{\tau}, x_{\tau}\right)$ around $\phi\left(c_{\tau-1}, x_{\tau-1}\right)$ gives

$$
\phi\left(c_{\tau}, x_{\tau}\right)=\phi\left(c_{\tau-1}, x_{\tau-1}\right)+\left(c_{\tau}-c_{\tau-1}\right) \theta_{c \tau}+\left(x_{\tau}-x_{\tau-1}\right) \theta_{x \tau}
$$

where $\theta_{c \tau}=(1 / 2) \partial \phi(c, x) / \partial c$ and $\theta_{x \tau}=(1 / 2) \partial \phi(c, x) / \partial x$, each evaluated at a level of $c$ between $c_{\tau}$ and $c_{\tau-1}$ and a level of $x$ between $x_{\tau}$ and $x_{\tau-1}$. Substituting this expression into the Euler equation and summing then gives

$$
\frac{c_{\tau}}{\tau}=\frac{\sum_{s=1}^{\tau} e_{s}}{\tau}
$$


where $e_{s}=\left[e_{s}^{*}+\left(b_{s}-1\right) \phi_{s}-\left(x_{s}-x_{s-1}\right) \theta_{x s}\right] / \theta_{c s}$ and $e_{1}=c_{1}$. This again yields approximate normality of log consumption,

$$
c_{\tau} \approx N\left(\tau \mu, \sigma^{2}\right)
$$

for some $\mu$ and $\sigma^{2}$, provided that a CLT can be applied to the average of the autocorrelated errors $e_{s}$.

\section{Detecting Departures from Log Normality}

We examine the closeness of observed data to log normality by comparing different features of the empirical distributions of log income and log expenditures to their theoretical normal counterparts. To visually depict departures from normality we construct quantile-quantile (QQ) plots as well as histograms of the sample, overlaid with a $N\left(\mu, \sigma^{2}\right)$ density function. The QQ plots are scatterplots of empirical quantiles against theoretical quantiles implied under normality. In particular, let $y_{(1)}<y_{(2)}<\cdots<y_{(n)}$ denote the ordered values of a sample of draws $y_{1}, y_{2}, \ldots, y_{n}$ of a random variable $Y$, and let $\Phi^{-1}$ denote the inverse of the cumulative distribution of a standard normal variable. The QQ plot depicts the points $\left\{y_{(i)}, \mu+\sigma \Phi^{-1}\left(\frac{i}{n}\right)\right\}$ for $i=1, \ldots, n$, which on average will lie along the 45-degree line if the data are independent draws of $Y$ having a normal $N\left(\mu, \sigma^{2}\right)$ distribution. We indicate the 5th, 25th, 50th, 75th and 95th quantile on each QQ plot.

To construct graphical comparisons or formal test statistics for normality requires estimation of the location and scale parameters $\mu$ and $\sigma$. Standard estimates of these and higher moments can be very sensitive to outliers, and both income and consumption data may well contain reporting errors, particularly at very low and very high income levels. We therefore use estimates and tests based on robust statistics, which mitigate the impact of gross errors and outliers in the data (see, e.g., Hampel et al., 1986). Consequently, in our application we will use the median $M(Y)$ and the population median absolute deviation $M A D(Y) \equiv M(|Y-M(Y)|)$ as our robust measures of location and scale. For normal distributions $M(Y)$ and $M A D(Y)$ are related to the mean and variance by $M(Y)=\mu$ and $M A D(Y) \simeq 0.6745 \sigma$ (where the appoximation $\simeq$ is just due to the number of decimal places used). The corresponding robust 
estimators of the location and scale parameters for a normal distribution are therefore

$$
\hat{\mu}=\hat{M}(Y), \quad \hat{\sigma}=\frac{M \hat{A} D(Y)}{0.6745} .
$$

where $\hat{M}(Y)$ and $M \hat{A} D(Y)$ denote the sample median and sample median absolute deviation. We provide histograms of the data, and superimposed on each histogram is a normal density function that uses these robust mean and variance estimates.

Given location and scale parameter estimates, tests for departure from normality can be implemented. We first construct Kolmogorov-Smirnov tests based on the distance between the empirical distributions of income and expenditure and the theoretical distributions obtained under normality. To account for estimation error in $\hat{\mu}$ and $\hat{\sigma}$, we obtained p-values for this test using 10,000 random samples generated under the null hypothesis of normality, $N(\hat{\mu}$, $\hat{\sigma}^{2}$ ), and counted the number of replicate samples that produced a test statistic greater than or equal to that calculated for the actual data.

We also construct two additional tests based on robust indicators of skewness and kurtosis. Hinkley (1975) and Groeneveld and Meeden (1984) suggest skewness measures of the form

$$
\frac{\left[Q_{1-p}(Y)-M(Y)\right]-\left[M(Y)-Q_{p}(Y)\right]}{Q_{1-p}(Y)-Q_{p}(Y)},
$$

where $Q_{\alpha}(Y)$ is the $\alpha$-th percentile of the distribution of $Y$. In our application we use quartile skewness, which takes $p=0.25$ and is zero for normal distributions. The resulting expression is analogous to estimating skewness by first using the median to center the data and scaling with the interquartile range. The numerator of (1) is then the difference of the positive distances between the upper half median and the median $\left(Q_{75}(Y)-M(Y)\right)$ and between the median and the lower half median $\left(M(Y)-Q_{25}(Y)\right)$, and the denominator is the sum of these distances. Positive (negative) values of this statistic indicate right (left) skewness. Additionally, this coefficient will take values in the interval $(-1,1)$, with $1(-1)$ representing extreme right (left) skewness.

Analogous to these other moments, for kurtosis we follow Moors (1988) and use

$$
\frac{\left[O_{7}(Y)-O_{5}(Y)\right]+\left[O_{3}(Y)-O_{1}(Y)\right]}{O_{6}(Y)-O_{2}(Y)},
$$

where $O_{\alpha}(Y)$ is the $\alpha$-th octile of the distribution of $Y$. This statistic is non-negative and not very sensitive (hence robust) to the extreme tails of the distribution, and for normal distributions it equals 1.233 (see Moors, 1988). 
We computed the sample analogues of both the skewness coefficient (1) and the kurtosis coefficient (2), and compare them to their theoretical values under the assumption of normality. P-values under the null hypothesis of normality were computed from 10,000 pseudo-samples as before.

\section{The Consumption and Income Data}

Most of our empirical analysis is based on expenditure and income data from the Consumer Expenditure (CEX) Interview Survey. The CEX is currently the only micro-level data set reporting comprehensive measures of consumption expenditures for a large cross-section of households in the United States. The Interview Survey has been collected continuously since 1980, with a sample selected on a rotating panel basis targeted at 5000 units each quarter until 1998, increasing about 30 percent after then.

Households are interviewed about their expenditures every three months over five consecutive quarters. Information is collected using recall questions on the usual weekly or monthly spending, depending on the item (see, e.g., Battistin 2003 for more details about the survey methodology). After the last interview households are dropped and replaced by a new unit, so that, by design, 20 percent of the sample changes every quarter. Expenditure information is collected in the second through the fifth interview; with one month recall expenditures are asked in the first interview only for bounding purposes. In the second and the fifth interview, the household's reference person is also asked about income in the previous twelve months. The information in the last interview can therefore be matched to the period covered by the expenditure data.

We used quarterly expenditures published by the US Bureau of Labor Statistics (BLS) between 1980 and 2003 to derive annual aggregate measures of expenditure at the household level. This information is contained in the Summary Expenditure Data section available in the public use data files. To this extent, we used only households who participated in the survey for all interviews (representing about 75-80 percent of the original sample) and sum their quarterly expenditures over the year covered by the four interviews. We considered the measure of total expenditure as published by the BLS after excluding 'cash contributions' and 'personal insurance and pensions', thus using a definition that includes expenditures for food, alcohol, 
housing, transportation, apparel, medical care, entertainment, and other miscellaneous items (such as personal care services, reading, education and tobacco products).For income, we use before tax figures as reported in the fifth interview by households who were classified as complete income reporters (though we checked that our results were largely unaffected when we also used income data from the second interview). Real income and expenditure figures were obtained throughout by deflating using the Consumer Price Index. Table 1 provides some sample summary statistics, including the cohort definitions and subsample sizes.

We complemented information from the CEX with information from the Panel Study of Income Dynamics (PSID) and from the British Family Expenditure Survey (FES) for the United Kingdom. Unlike the CEX, the PSID collects longitudinal annual data on a sample of households followed on a consistent basis since 1968. We examine family disposable income in the PSID for a sample of couples with and without children as described in Blundell, Pistaferri and Preston (2004, 2005). The UK FES contains both detailed household income and consumption data within the same survey, though total expenditures are only recorded for a few weeks, and so may suffer from measurement errors due to problems like infrequency of purchase.

For all surveys, for stability we focus on a sample of married couples (with or without children) and define cohorts based on the year of birth of the head, which we conventionally take to be the husband. Tables 2 and 3 present the summary statistics for our PSID and the FES samples, respectively. A caveat regarding these data is that there is likely to be under reporting in both tails of the income and consumption distributions, because many poor households (such as the homeless) will be excluded, and wealthy households are also likely to be underreported, both because their incentive to fill out the lengthy surveys is low, and because of data topcoding by the reporting agencies. This is one reason why we used robust measures distribution moments as discussed earlier.

\section{The Empirical Distributions of Consumption and In- come}

The Euler equation and permanent income models of the previous sections are likely to be oversimplifications of reality. Our goal here is to check if their theoretical distribution im- 
plications are roughly consistent with empirical distributions of income and consumption data, which would be the case if the models at least coarsely approximate the income and consumption behavior of most households.

To assess empirically the distribution implications of these models, consider a cross-section of individuals, all of the same age $\tau$. By definition, $\tau \mu$ and $\sigma^{2}$ are unconditional moments of the distribution of consumption. If the individuals in the sample are sufficiently similar, in the sense of having similar unconditional moments $\mu$ and $\sigma^{2}$, then the model can be tested by examining whether the shape of the distribution of $c_{\tau}$ across individuals in the sample is close to normal. These are unconditional moments for each individual, so these tests do not rule out conditional differences or correlations. For example, shocks can be conditionally heteroskedastic and correlated across individuals. Similarly, having $\mu$ and $\sigma^{2}$ be the same across consumers does not mean that every consumer has the same consumption or the same permanent income on average, but rather that each individual's age $\tau$ permanent income or consumption is drawn from some common underlying unconditional distribution that is characterized by these moments, where by 'unconditional' we mean not conditioning on the individual's previous consumption, income, or other attributes.

Figures 4-7 show the distribution of log expenditure and log income across the life-cycle for each of four birth decade cohorts available from the Consumer Expenditure Surveys, beginning with the youngest cohort (those born in the 1960's). For each cohort, the distribution of log consumption and log income is presented at each five year age interval in their lifecycle. So, e.g., Figures 4(a) and 4(b) are consumption and income from the 1960's cohort when they were aged 26-30, Figures $4(\mathrm{c})$ and $4(\mathrm{~d})$ are the same variables for the same cohort when they are aged 31-35, Figure 5 has the same data for the 1970's cohort going up to age 41-45, etc.,.

Figure 4(a) shows a log real expenditure distribution that is very close to normal. In contrast, Figure 4(b) shows that log real income for these households is much further from normal with the upper tail skewness that is typical of income distributions, and greater kurtosis as well. A similar pattern holds across all age groups. These and the other log income distributions we report also show a long lower tail. We expect that at least some of this observed lower tail behavior is due to measurement error, e.g., there may be considerable under reporting of income at these levels, and relatively small absolute errors in reported 
income at low absolute levels of income may cause large distortions in the distribution of income after taking logs.

Comparing people of the same ages across cohorts in Figures 4 to 7 shows that younger cohorts have a higher dispersion of income and consumption, e.g., 31 to 35 year olds born in the 1960's have a higher variance of income than 31 to 35 year olds born in the 1970's. A similar pattern holds up across most age groups and cohorts. Also, in accordance with Kalecki (1945) and Deaton and Paxson (1992), and consistent with Gibrat's law, as every birth cohort ages, their distributions of income and consumption become more dispersed.

In Figure 8 we report data for the youngest available age group, which is 21-25 year olds born in the 1960's. Figure 8 shows that the distributions for these very young households are further from log normal than for the older groups, which is again consistent with our theory of distributions determined by Gibrat's law. Above ages 25 or 30 departures from log normality of consumption are very small and do not seem to systematically decrease further with age, which suggests that by relatively early in one's working life enough shocks have accumulated to get close to asymptotic normality.

Our theory suggests that consumption should be closer to log normal than income, because income contains a potentially large transitory component in addition to a log normal permanent income component. This is what we found in the CEX, but one might worry that departures from log normality in CEX income data could be due measurement error, because income may be measured less precisely than consumption in that data set. As a check, in Figure 9 we examine income by birth cohort and age but this time for log family disposable income from the PSID data set, which measures income more carefully than the CEX. We find significant deviations from normality of log income in this data, similar to the departures from log normality found in the CEX.

To show that the finding of normality of log consumption is not exclusive to the United States, in Figure 10 we report consumption data by birth cohort and age from the British FES. As in the CEX, consumption in the FES is very close to log normal.

Our data includes households with varying numbers of children, because subpopulations sorted by household size would not be comparable across age brackets. For example, households at age 40 with three children are more representative of the general population than 
households at age 20 that have three children. However, numbers of children correlates with income, and affects the propensity to consume out of current income. So as further check on the robustness of our results, we recalculated distributions after dividing each household's income and consumption by $\sqrt{n}$ where $n$ is family size, thereby following a common practice of using $\sqrt{n}$ as an equivalence scale. These results, which remain consistent with our other findings, are presented in Figure 11.

\section{Conclusions}

The income distribution has long been known to be approximately log normal. We have shown that the consumption distribution is also close to log normal, and that within demographically homogeneous groups, the distribution of consumption is much closer to log normal than is the distribution of income. We also demonstrate that these empirical regularities are implications of traditional models of the evolution of income and consumption, specifically, that the theory which motivates Gibrat's law should apply to permanent income and consumption (via Euler equations), rather than to total income as originally formulated.

We would not expect perfect normality for a variety of reasons. Traditional permanent income and Euler equation models are implausibly simplistic, so we should not expect them to hold exactly. Also, the CLT is an asymptotic property while individuals only have finite lifespans. Even when permanent income is close to log normal for some individuals, their consumption may depart from log normality if marginal utility differs substantially from log consumption, or if liquidity constraints, precautionary savings, or purchases of large durables produce enough dependence in Euler equation innovations to violate the conditions required for a CLT. More generally, normality may not hold for some individuals because their time series of shocks may possess features such as $b_{\tau}$ 's far from one or long memory, that violate the regularity conditions required for a CLT. Despite these possible problems, we find that the observed distributions of consumption and income to be broadly consistent with the distribution implications of these models, across cohorts, over time, and across data sets.

Other explanations for the observed consumption and income distributions may exist. For example, if consumption is very badly measured, then its observed distribution could be dominated by measurement errors that happen to be log normal. Another possibility is 
based on the observation that higher income households tend to consume a smaller fraction of income than lower income households, resulting in a consumption distribution that has a thinner upper tail than the income distribution. If the income distribution is close to log normal except for a thick (Pareto) upper tail, the consumption distribution should then have a thinner upper tail, which could by coincidence be almost the same size as its lower tail, resulting in a near normal distribution. These alternative explanations for consumption log normality require coincidences that we find less plausible than our derivations based on permanent income and Euler equation models, though these alternatives could be contributing factors in the observed distributions.

As discussed in the introduction, the finding that Gibrat's law applies to consumption within cohorts has many important implications for welfare and inequality measurement, aggregation, and econometric model analysis, and results in additional regularities in the distributions of related variables. It would be interesting to test if other economic variables that are determined either by Euler equations or decompositions into permanent and transitory components display a similar conformity to Gibrat's law.

\section{References}

[1] Aitchison, J., and J.A.C. Brown (1957), The Lognormal Distribution, Cambridge University Press, Cambridge.

[2] Banks, J. Blundell, R. and A. Lewbel (1997), 'Quadratic Engel Curves, Indirect Tax Reform and Welfare', Review of Economics and Statistics, Vol. LXXIX, No.4, 527-539, November.

[3] Battistin, E. (2003), "Errors in Survey Reports of Consumption Expenditures", Working Paper 07/03, Institute for Fiscal Studies, London.

[4] Blundell, R., L. Pistaferri and I. Preston (2004), "Imputing consumption in the PSID using food demand estimates from the CEX", Institute for Fiscal Studies, WP04/27, (http://www.ifs.org.uk/ workingpapers/ wp0427.pdf).

[5] Blundell, R., L. Pistaferri and I. Preston (2005), "Consumption Inequality and Partial Insurance", Consumption Inequality and Partial Insurance, (with L. Pistaferri and I. Preston), Institute for Fiscal Studies, WP04/27, revised May 2005.

[6] Blundell, R., and I. Preston (1995), 'Income, Expenditure and the Living Standards of UK Households' Fiscal Studies, Vol. 16, No.3, 40-54, 1995. 
[7] Blundell, R., and I. Preston (1998), "Consumption inequality and income uncertainty", Quarterly Journal of Economics 113, 603-640.

[8] Deaton, A. (1992), Understanding Consumption. Baltimore: John Hopkins University Press.

[9] Deaton, A., and C. Paxson (1994), "Intertemporal choice and inequality", Journal of Political Economy, 102, 384-94.

[10] Friedman, M. (1957), A Theory of the Consumption Function, Princeton: Princeton University Press.

[11] Gabaix, X. (1999) 'Zipf's Law for Cities: An Explanation', The Quarterly Journal of Economics, vol. 113, no. 3 (August), pp. 739-767.

[12] Gibrat, R. (1931). Les Inegalites Economiques. Librairie du Recueil Sirey. Paris.

[13] Groeneveld, R.A. and Meeden, G. (1984), Measuring Skewness and Kurtosis, The Statistician, Vol. 33, No. 4, 391-399.

[14] Hall, R. E. (1978), "Stochastic Implications of the Life Cycle-Permanent Income Hypothesis: Theory and Evidence," The Journal of Political Economy, 86, 971-987.

[15] Hampel, F.R., Ronchetti, E.M., Rousseeuw, P.J., and Stahel, W.A. (1986), Robust Statistics, John Wiley and Sons: New York.

[16] Hinkley, D.V. (1975), On Power Transormations to Simmetry, Biometrika, Vol. 62, No. $1,101-111$.

[17] Kalecki, M. (1945), "On the Gibrat Distribution," Econometrica, 13, 161-170.

[18] MaCurdy, T. E. (1982), "The use of time series processes to model the error structure of earnings in a longitudinal data analysis," Journal of Econometrics, 18, 83-114.

[19] Meghir, C., and L. Pistaferri (2004), "Income variance dynamics and heterogeneity", Econometrica, 72(1), 1-32.

[20] Meyer, B. and J. Sullivan (2003), "Measuring the Well-Being of the Poor Using Income and Consumption', Journal of Human Resources, 1180-220.

[21] Moors, J.J.A. (1988), A Quantile Alternative to Kurtosis, The Statistician, Vol. 37, No. $1,25-32$.

[22] Wooldridge, J. M. and H White, (1988), "Some Invariance Principles and Central Limit Theorems for Dependent Heterogeneous Processes," Econometric Theory, 4, 210-230.

[23] Zipf, G.K. (1949), Human Behavior and the Principle of Least Effort, Addison-Wesley. 
Figure 1: The Distribution of Log Household Income in the US

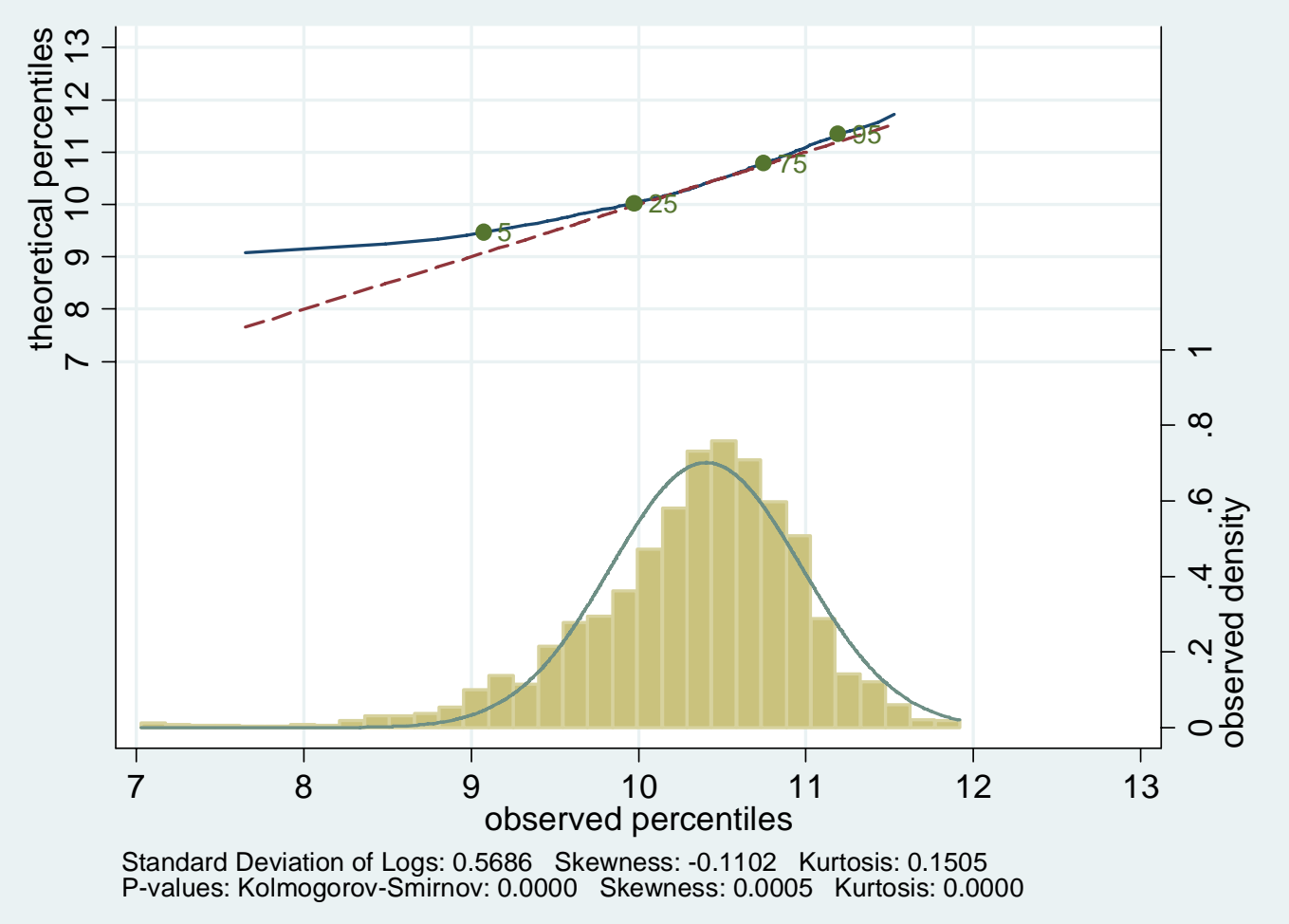

Notes: Age 31-35, income

Figure 2: The Distribution of Log Household Consumption in the US

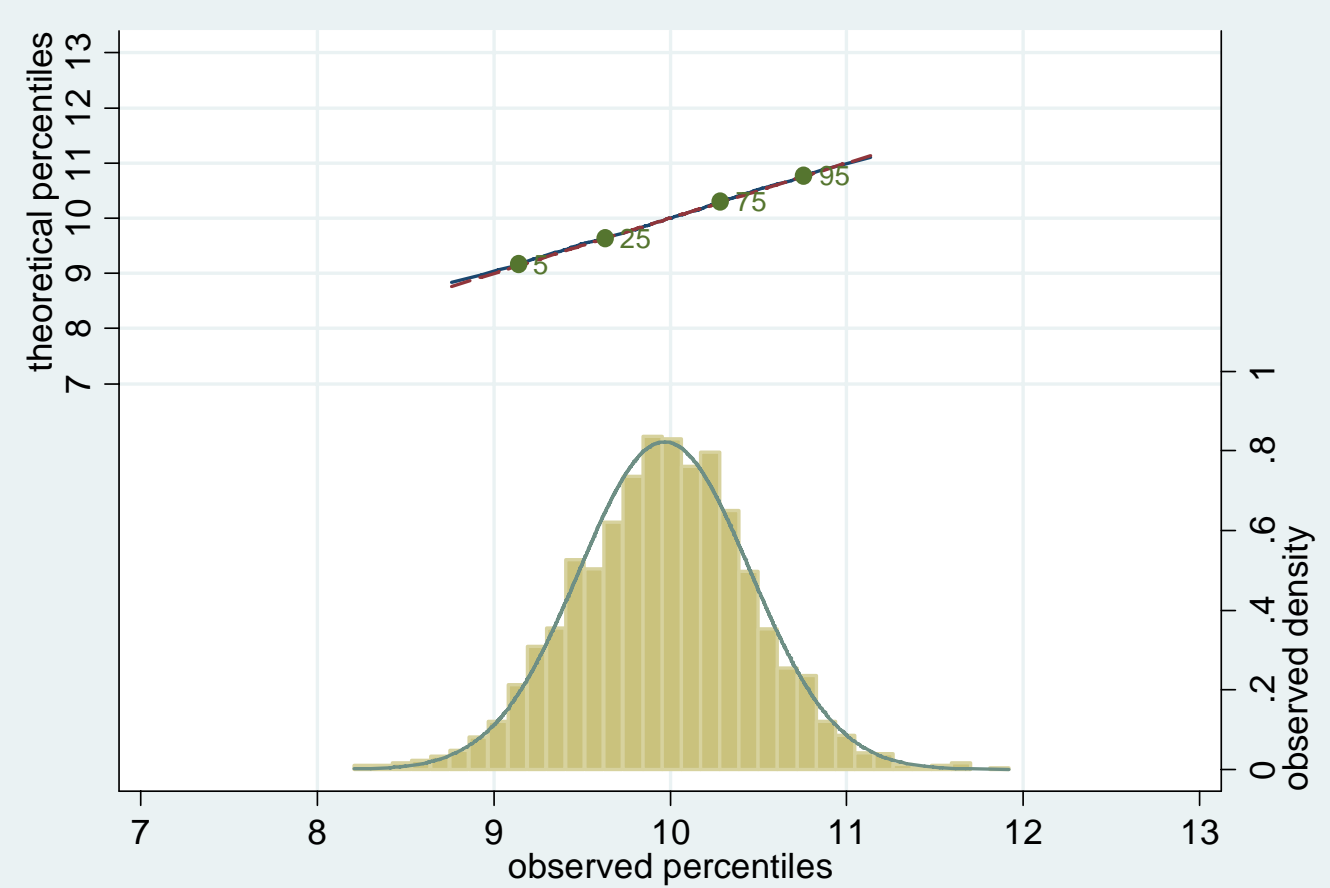

Standard Deviation of Logs: 0.4848 Skewness: -0.0266 Kurtosis: 0.0117

P-values: Kolmogorov-Smirnov: 0.3580 Skewness: 0.3843 Kurtosis: 0.7203

Notes: Age 31-35, expenditure 
Figure 3: Consumption in the UK

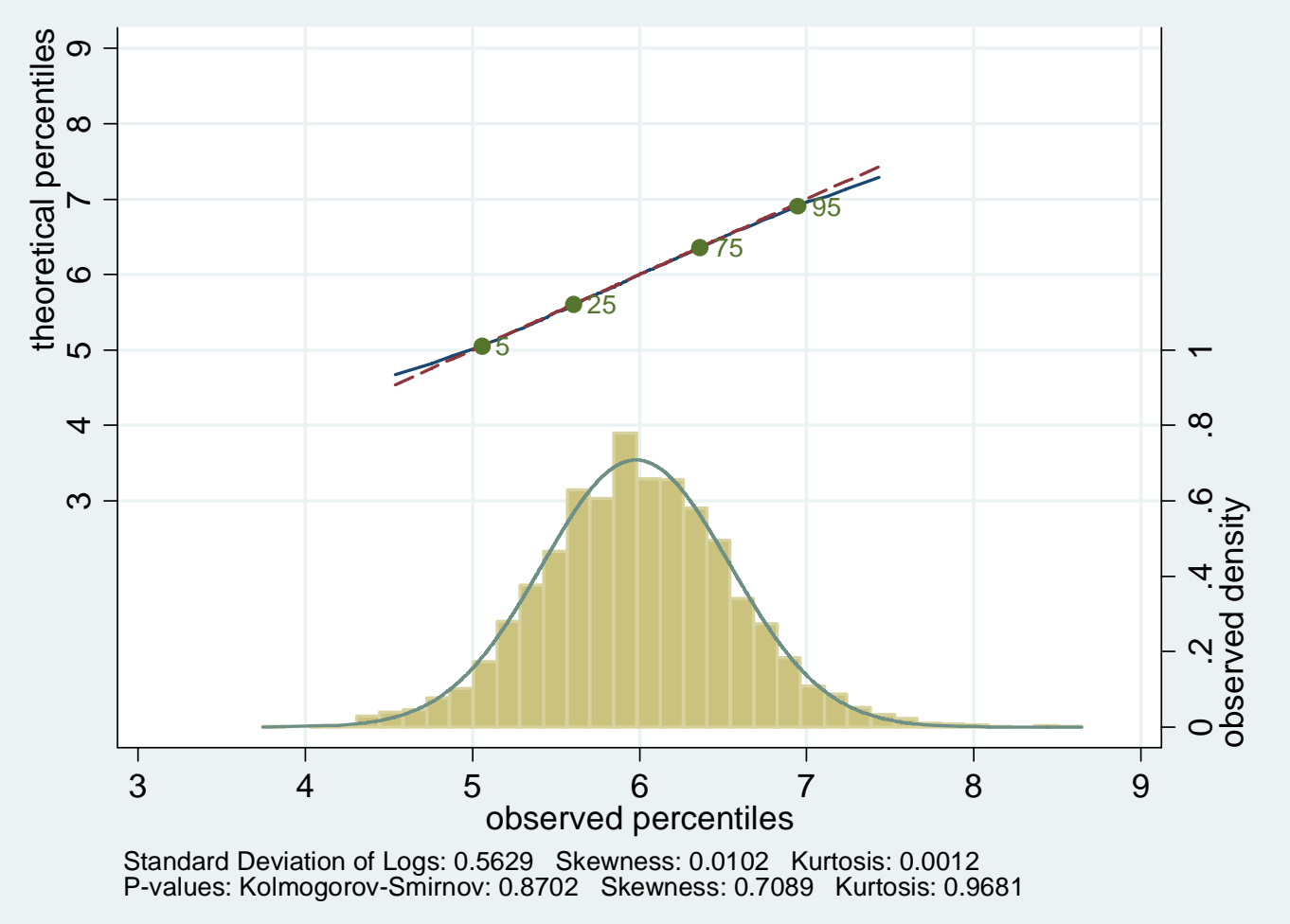

Notes: FES EXP DATA: COHORT 1930-39, AGE 51-55 
Table 1: CEX data (1986-2002)

4 cohorts by year of birth: 1930-39, 1940-49,1950-59, 1960-69

3 groups by interview year: 1986-90, 1991-95, 1996-2000

Sample size:

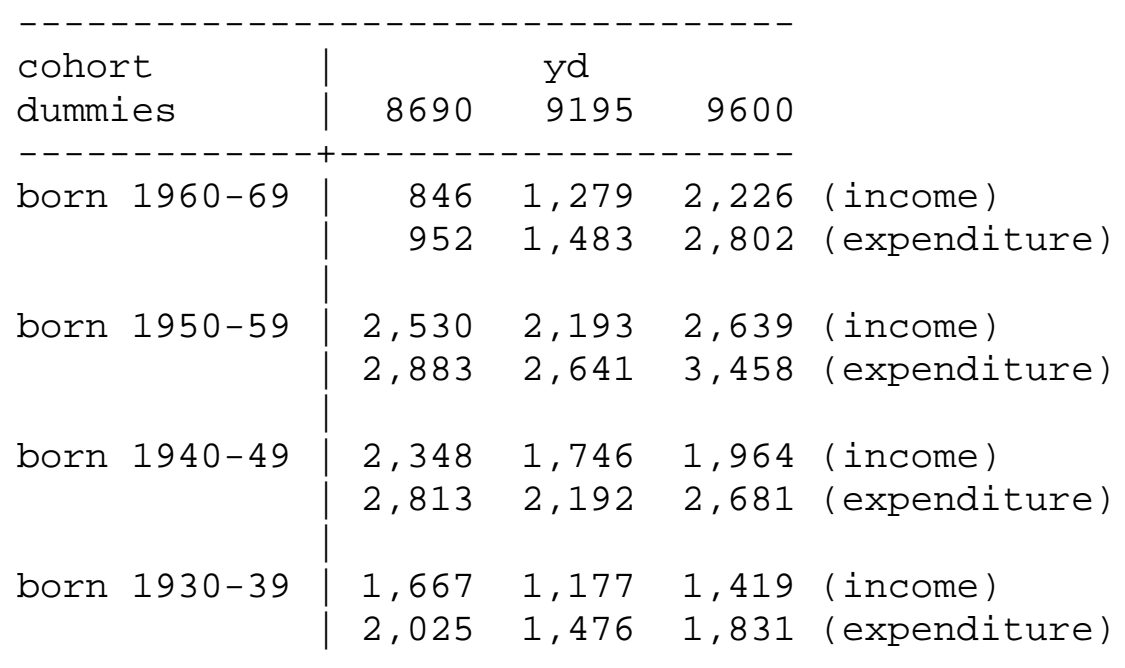

Reference age:

\begin{tabular}{lccc}
\multicolumn{1}{c}{ Year } & 8690 & 9195 & 9600 \\
Cohort & & & \\
$1960-69$ & $21-25$ & $26-30$ & $31-35$ \\
$1950-59$ & $31-35$ & $36-40$ & $41-45$ \\
$1940-49$ & $41-45$ & $46-50$ & $51-55$ \\
$1930-39$ & $51-55$ & $56-60$ & $61-65$
\end{tabular}

Notes: Expenditure: total expenditure as published by the BLS (excluding "cash contributions" and "personal insurance and pensions"): Income: total family income btax for complete income reporters only $-2^{\text {nd }}$ interview CEX (same story by considering the $5^{\text {th }}$ interview). 
Table 2: PSID data (from BPP paper: 1967-1992)

\begin{tabular}{r|r|r}
$\begin{array}{r}\text { cohort } \\
\text { dummies }\end{array}$ & \multicolumn{1}{|c}{ yd } \\
$8690 \mid$ & Total \\
\hline born $1950-59$ & $10,164 \mid$ & 10,164 \\
born 1940-49 & 5,642 & 5,642 \\
born 1930-39 & $3,366 \mid$ & 3,366 \\
\hline & \multicolumn{3}{c}{ Total | $19,172 \mid$} & 19,172
\end{tabular}

Reference age:

$\begin{array}{ll}\quad \text { Year } & 8690 \\ \text { Cohort } & \\ 1950-59 & 31-35 \\ 1940-49 & 41-45 \\ 1930-39 & 51-55\end{array}$

Notes: Income: total family income as in Blundell, Pistaferri and Preston (2004).

Table 3: FES data (1974-2000)

\begin{tabular}{l|lll}
$\begin{array}{l}\text { cohort } \\
\text { dummies }\end{array}$ & \multicolumn{3}{|c}{ yd } \\
\hdashline-1690 & 9195 & 9600 \\
\hline born 1950-59 & 4,973 & 4,682 & 4,111 \\
born 1940-49 & 4,828 & 4,171 & 3,568 \\
born 1930-39 & 3,587 & 3,038 & 2,633
\end{tabular}

Notes: Expenditure; definition as for the CEX 
Figure 4: The 1960-69 Birth Cohort from the CEX

(a) Age 26-30, expenditure

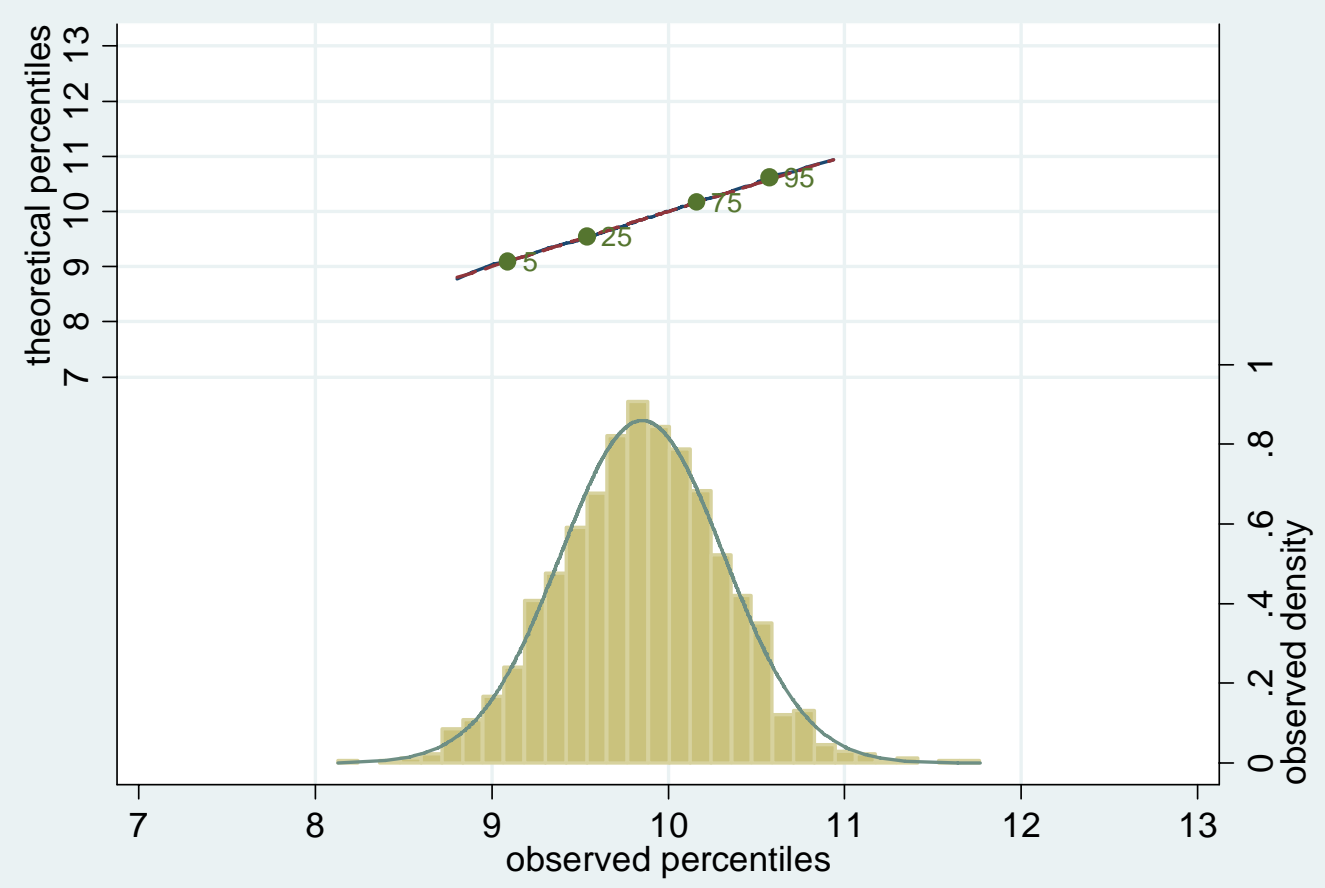

Standard Deviation of Logs: 0.4640 Skewness: -0.0074 Kurtosis: 0.0120

P-values: Kolmogorov-Smirnov: 0.8999 Skewness: 0.8655 Kurtosis: 0.8020

\section{(b) Age 26-30, income}

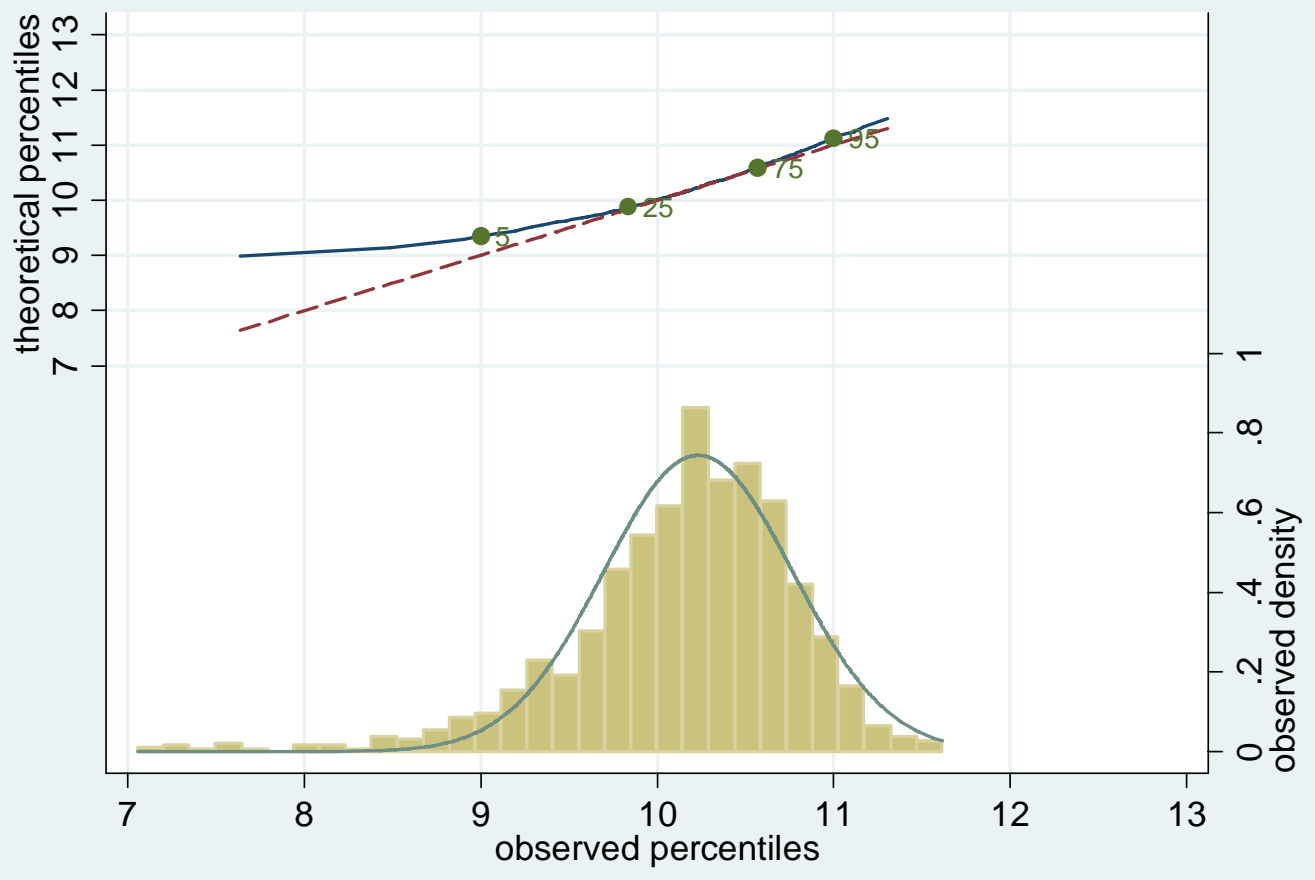

Standard Deviation of Logs: 0.5364 Skewness: -0.0787 Kurtosis: 0.1229

P-values: Kolmogorov-Smirnov: 0.0007 Skewness: 0.0871 Kurtosis: 0.0144 
(c) Age 31-35, expenditure

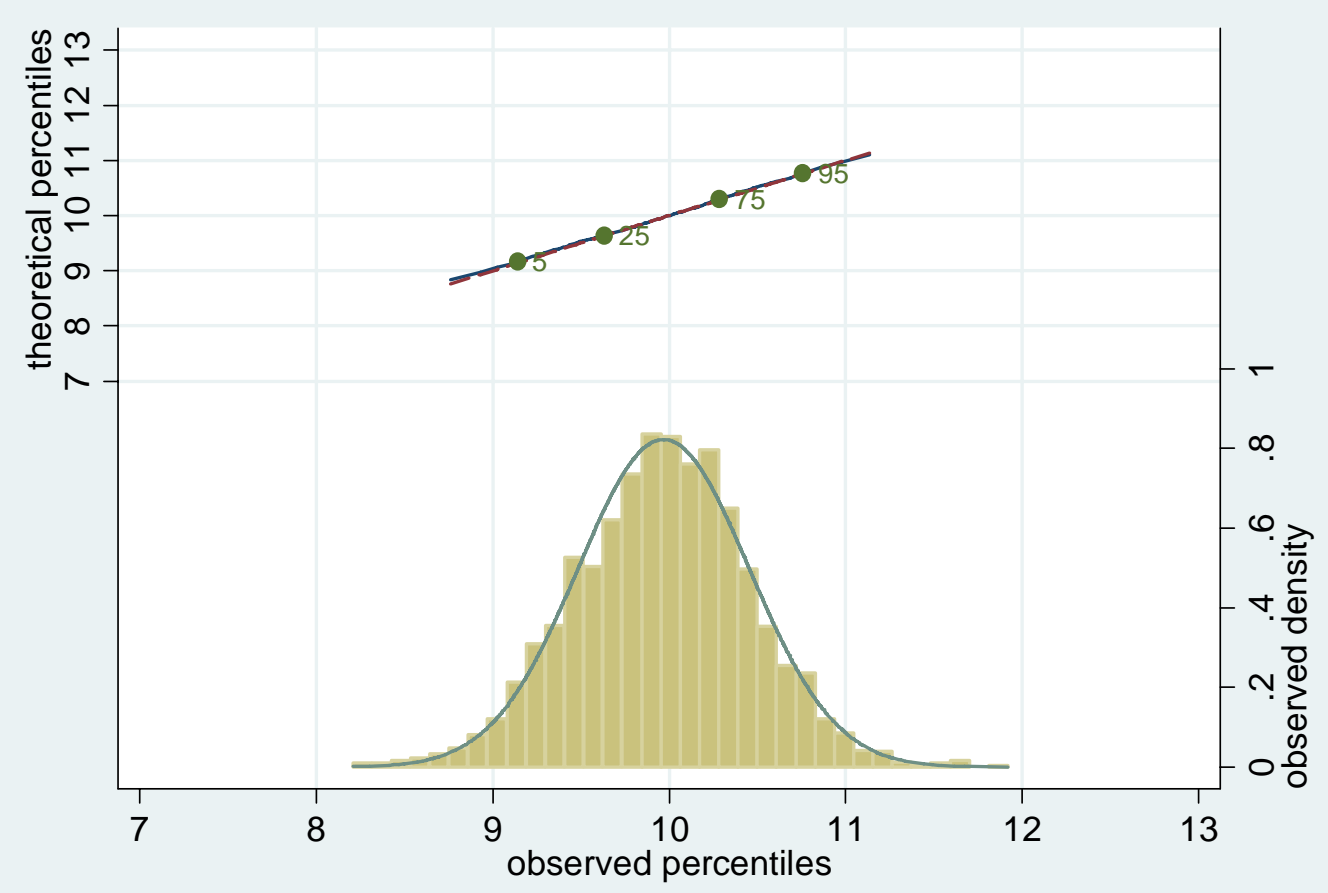

Standard Deviation of Logs: 0.4848 Skewness: -0.0266 Kurtosis: 0.0117

P-values: Kolmogorov-Smirnov: 0.3580 Skewness: 0.3843 Kurtosis: 0.7203

\section{(d) Age 31-35, income}

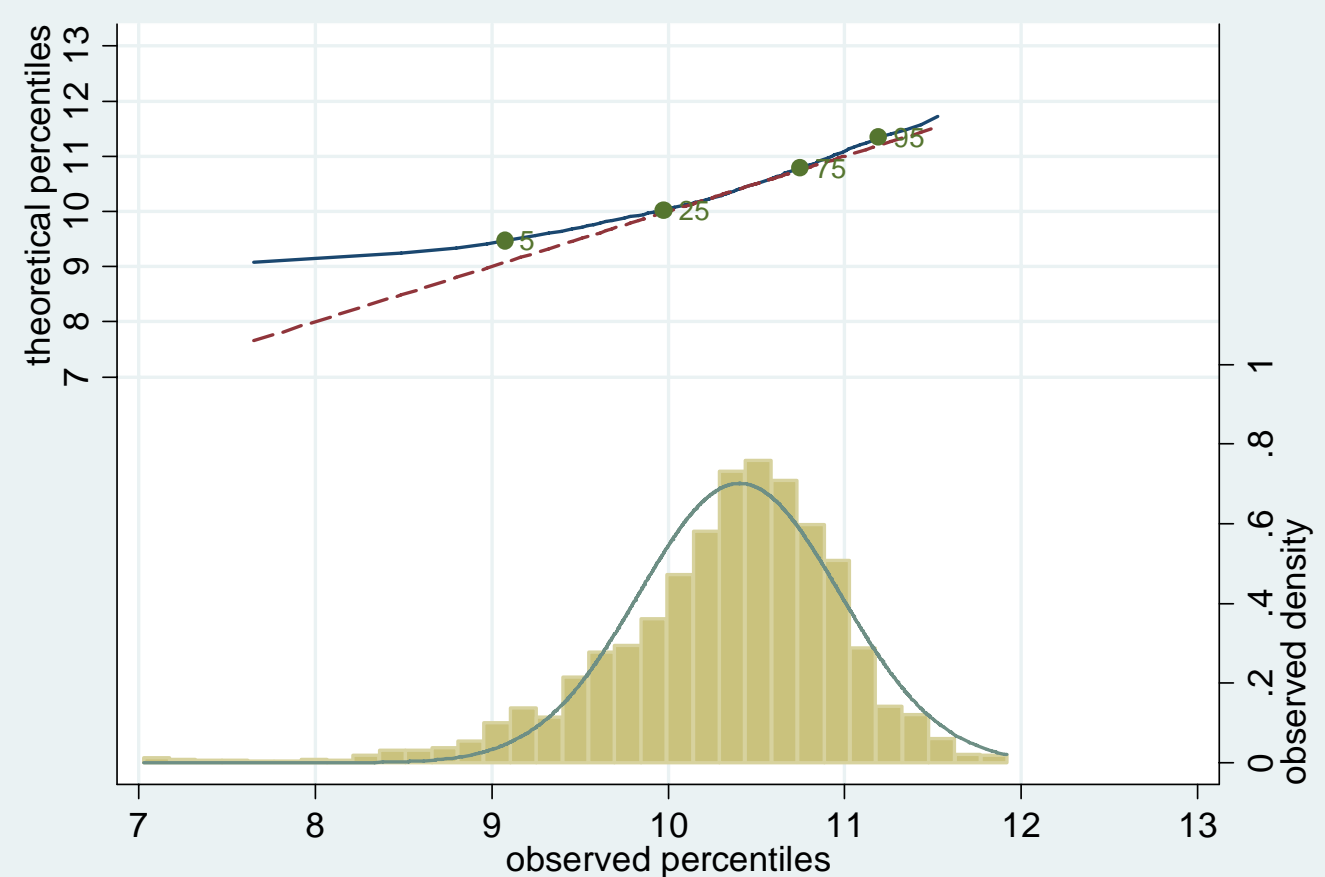

Standard Deviation of Logs: 0.5686 Skewness: -0.1102 Kurtosis: 0.1505

P-values: Kolmogorov-Smirnov: 0.0000 Skewness: 0.0005 Kurtosis: 0.0000 
Figure 5: The 1950-59 Birth Cohort from the CEX

\section{(a) Age 31-35, expenditure}

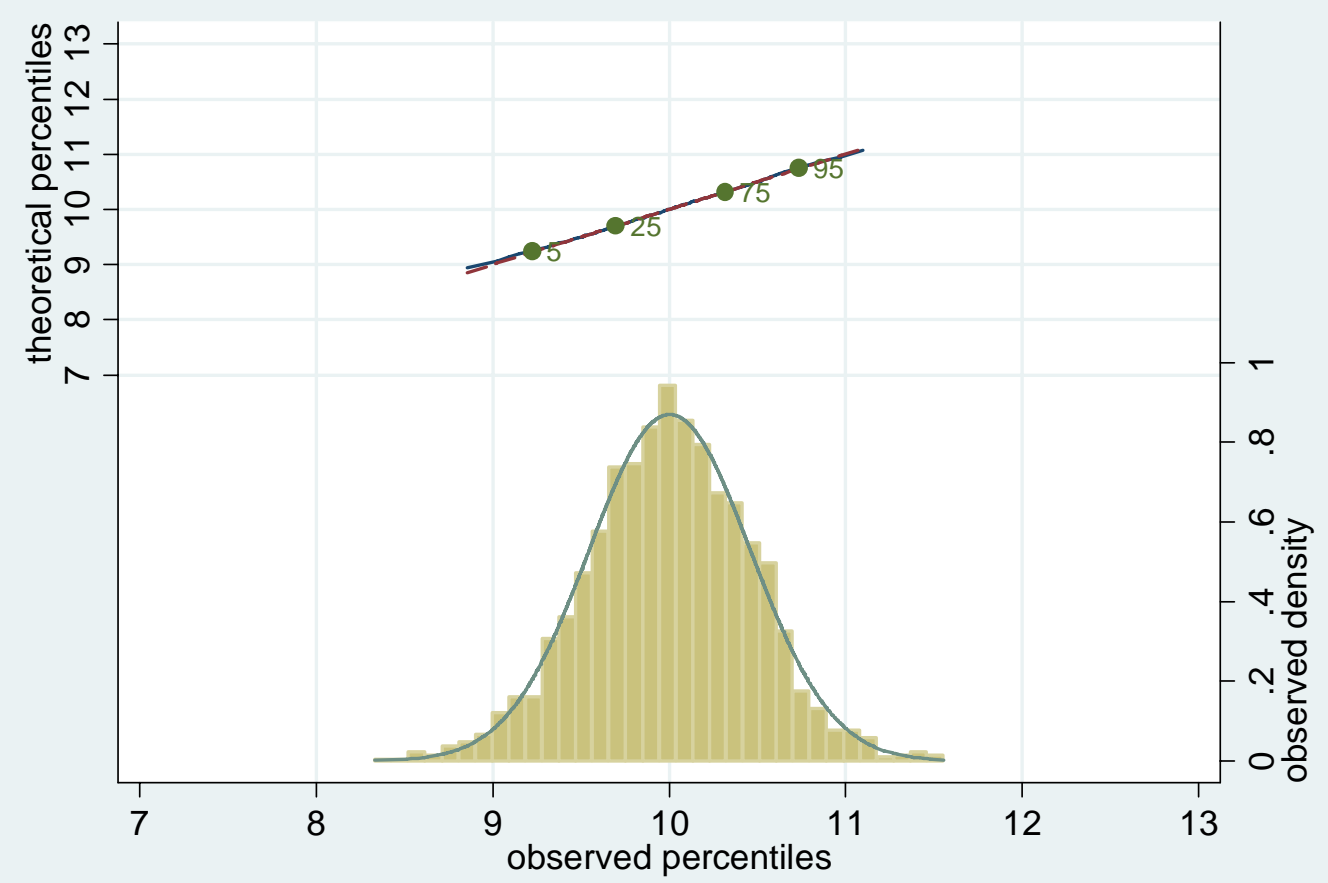

Standard Deviation of Logs: 0.4586 Skewness: 0.0122 Kurtosis: 0.0198

P-values: Kolmogorov-Smirnov: 0.9431 Skewness: 0.6917 Kurtosis: 0.5394

\section{Age 31-35, income}

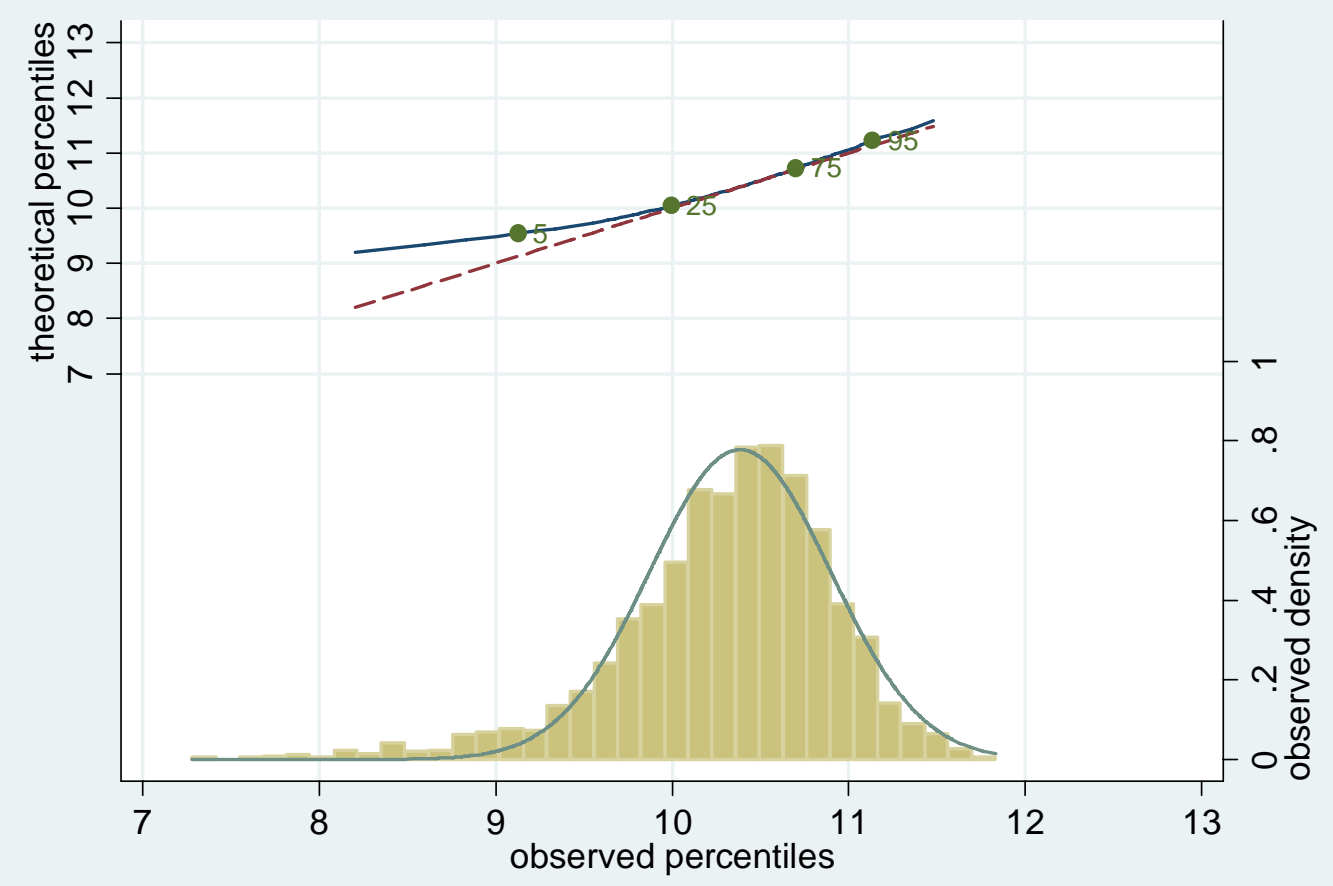

Standard Deviation of Logs: 0.5129 Skewness: -0.1011 Kurtosis: 0.0621

P-values: Kolmogorov-Smirnov: 0.0000 Skewness: 0.0013 Kurtosis: 0.0762 


\section{Age 36-40, expenditure}

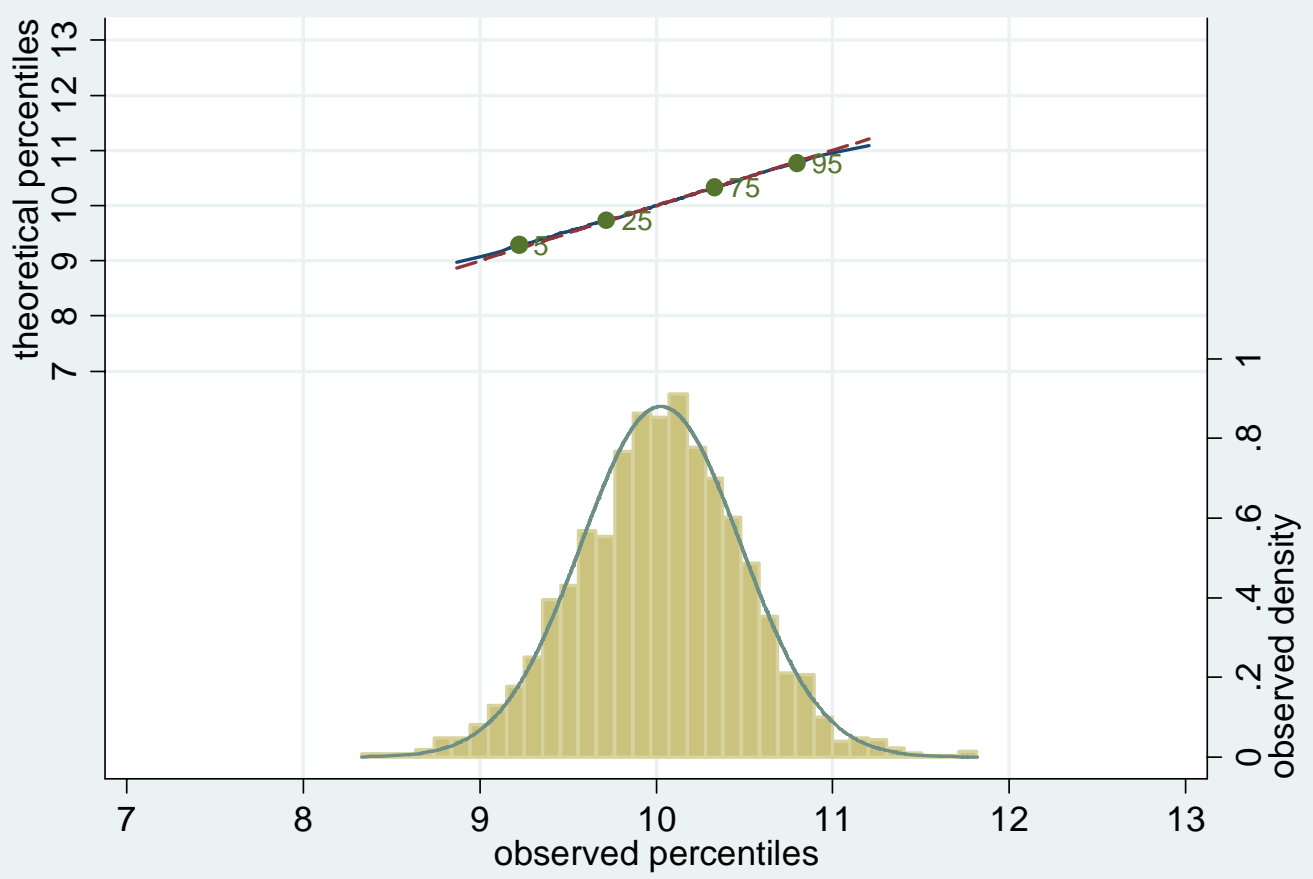

Standard Deviation of Logs: 0.4532 Skewness: 0.0002 Kurtosis: 0.0669

P-values: Kolmogorov-Smirnov: 0.3343 Skewness: 0.9954 Kurtosis: 0.0505

\section{Age 36-40, income}

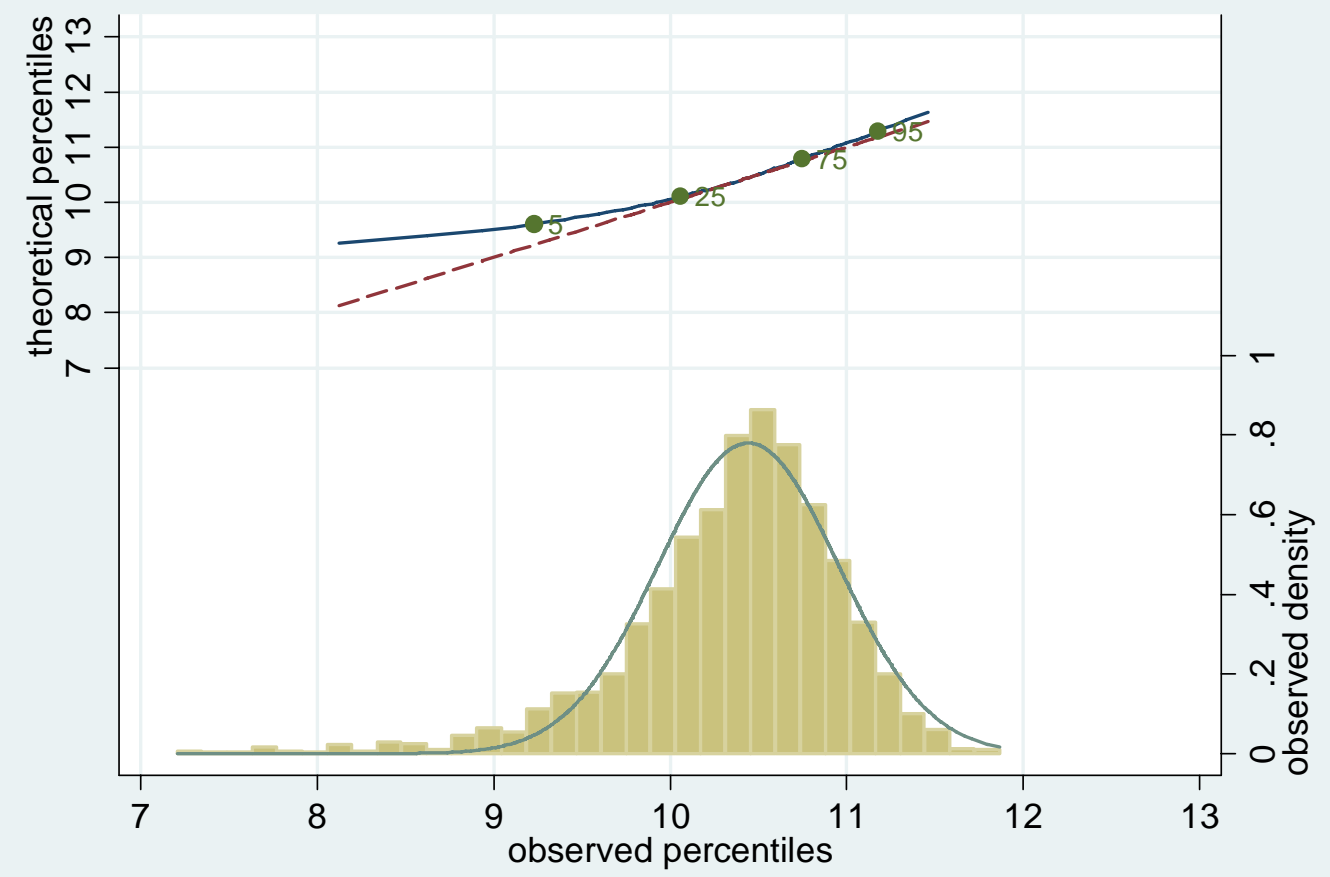

Standard Deviation of Logs: 0.5115 Skewness: -0.1139 Kurtosis: 0.1199

P-values: Kolmogorov-Smirnov: 0.0000 Skewness: 0.0002 Kurtosis: 0.0016 


\section{Age 41-45, expenditure}

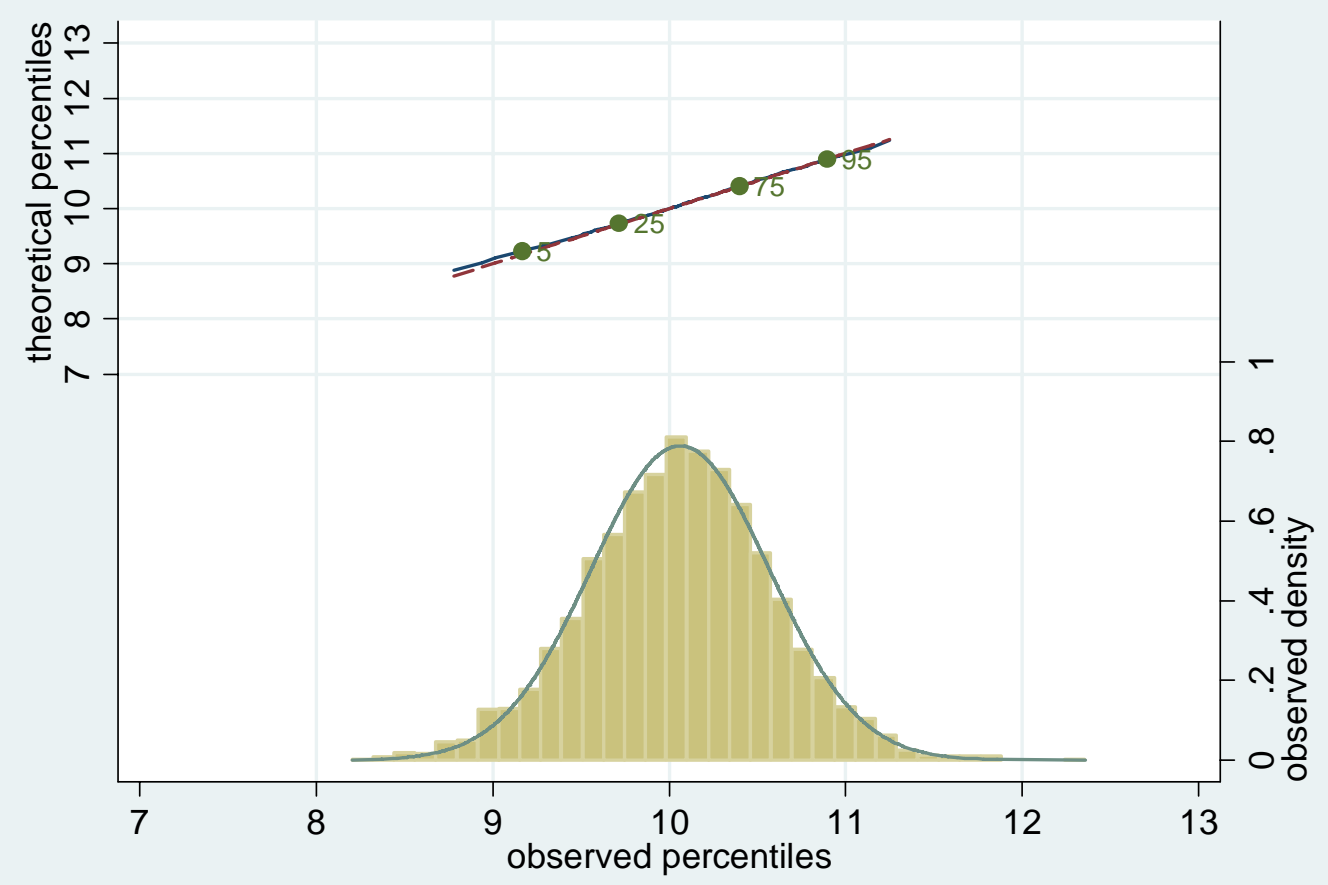

Standard Deviation of Logs: 0.5055 Skewness: -0.0180 Kurtosis: 0.0222

P-values: Kolmogorov-Smirnov: 0.5778 Skewness: 0.5123 Kurtosis: 0.4622

\section{Age 41-45, income}

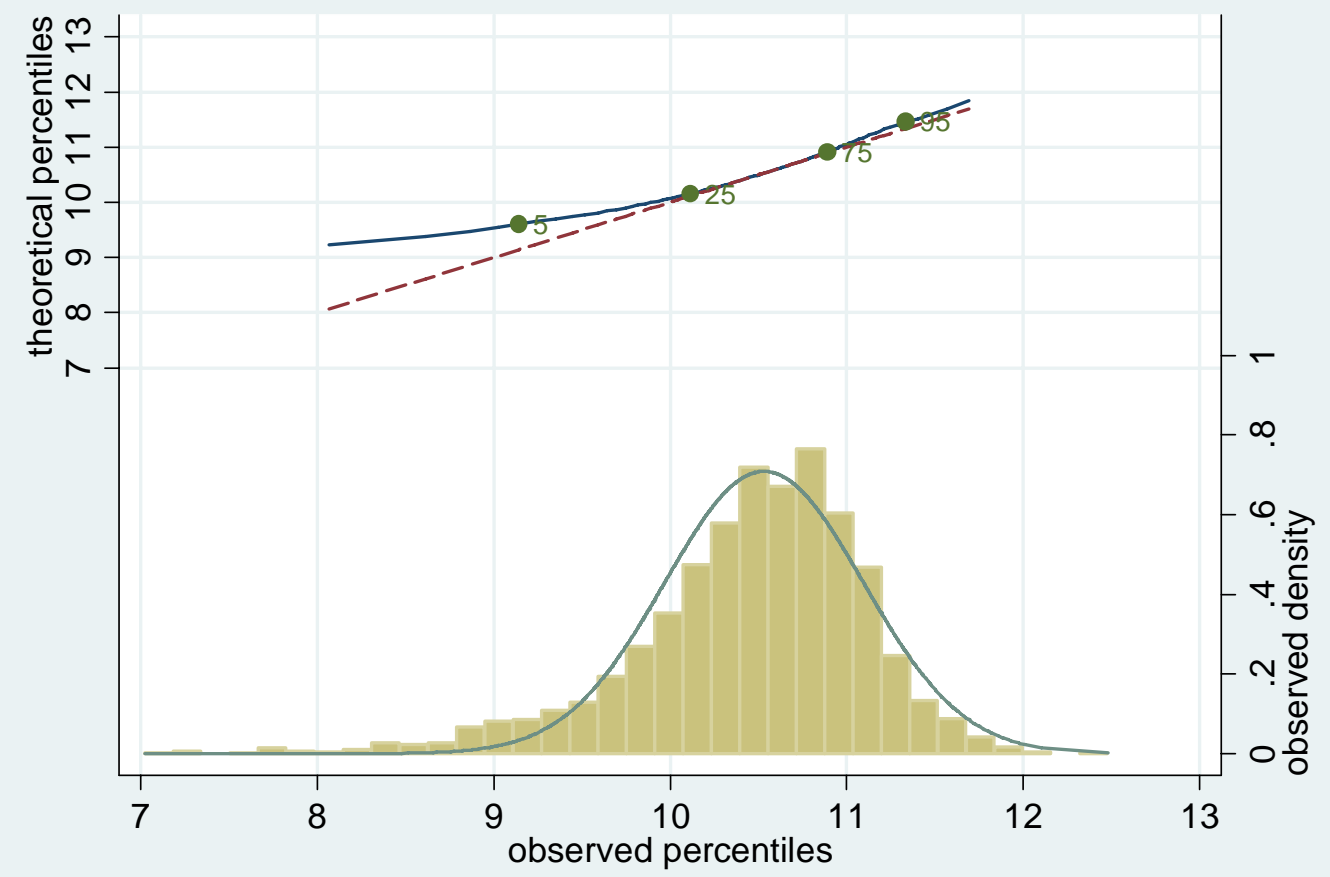

Standard Deviation of Logs: 0.5629 Skewness: -0.0783 Kurtosis: 0.0787

P-values: Kolmogorov-Smirnov: 0.0000 Skewness: 0.0140 Kurtosis: 0.0248 
Figure 6: The 1940-49 Birth Cohort from the CEX

\section{Age 41-45, expenditure}

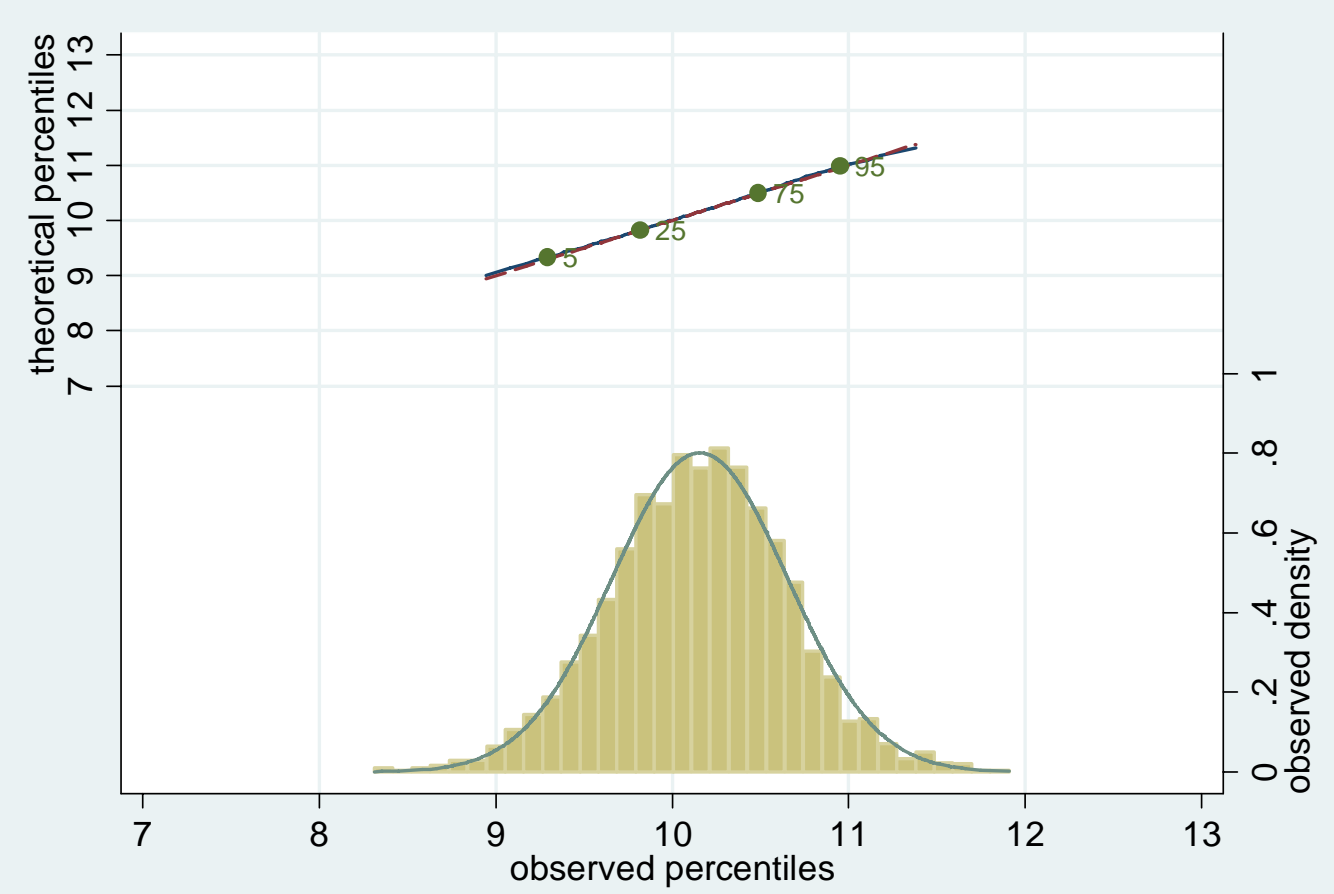

Standard Deviation of Logs: 0.4980 Skewness: -0.0081 Kurtosis: -0.0205

P-values: Kolmogorov-Smirnov: 0.7181 Skewness: 0.7931 Kurtosis: 0.5307

\section{Age 41-45, income}

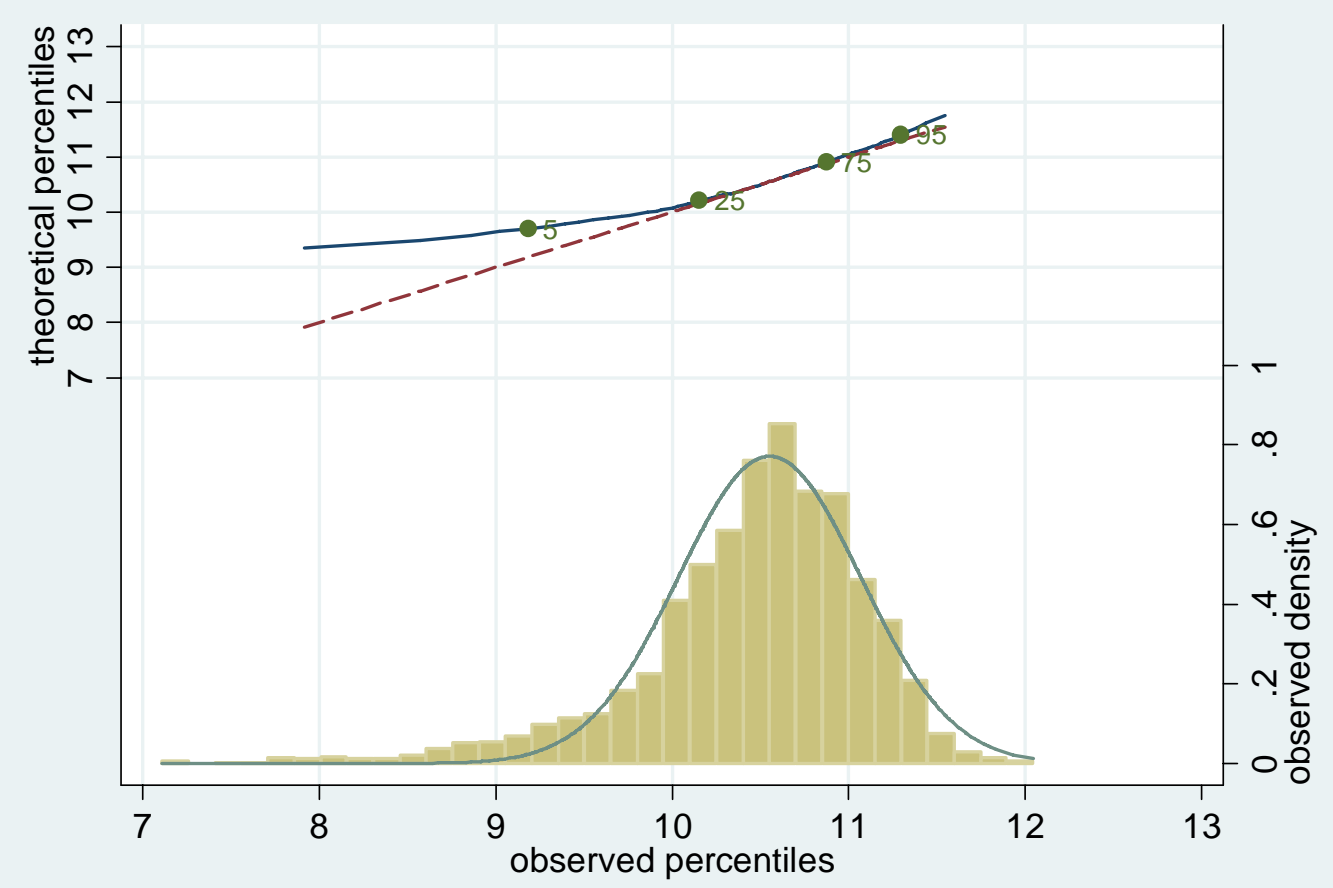

Standard Deviation of Logs: 0.5172 Skewness: -0.1061 Kurtosis: 0.1526

P-values: Kolmogorov-Smirnov: 0.0000 Skewness: 0.0007 Kurtosis: 0.0000 


\section{Age 46-50, expenditure}

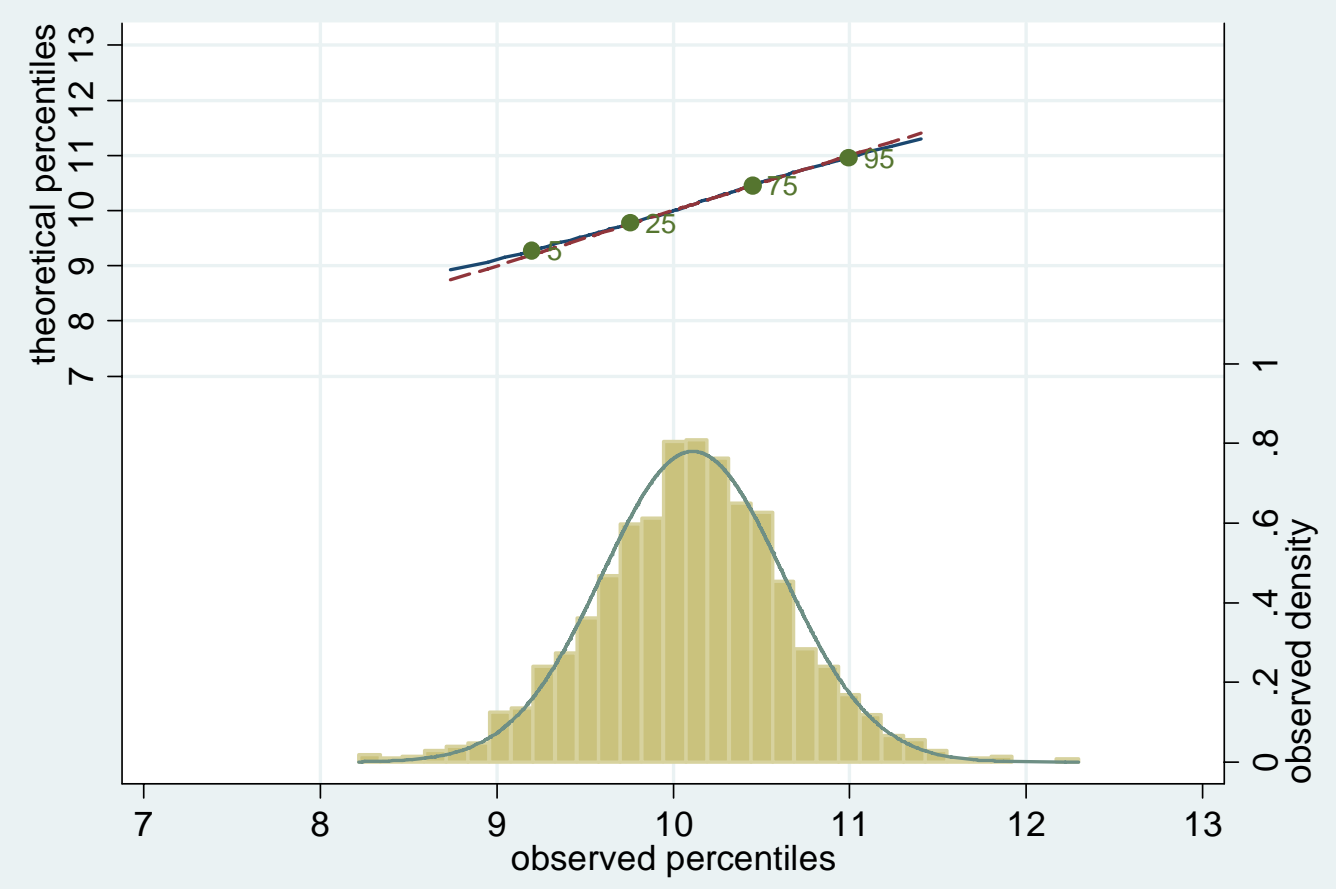

Standard Deviation of Logs: 0.5113 Skewness: -0.0196 Kurtosis: 0.0496

P-values: Kolmogorov-Smirnov: 0.3090 Skewness: 0.5807 Kurtosis: 0.1896

\section{Age 46-50, income}

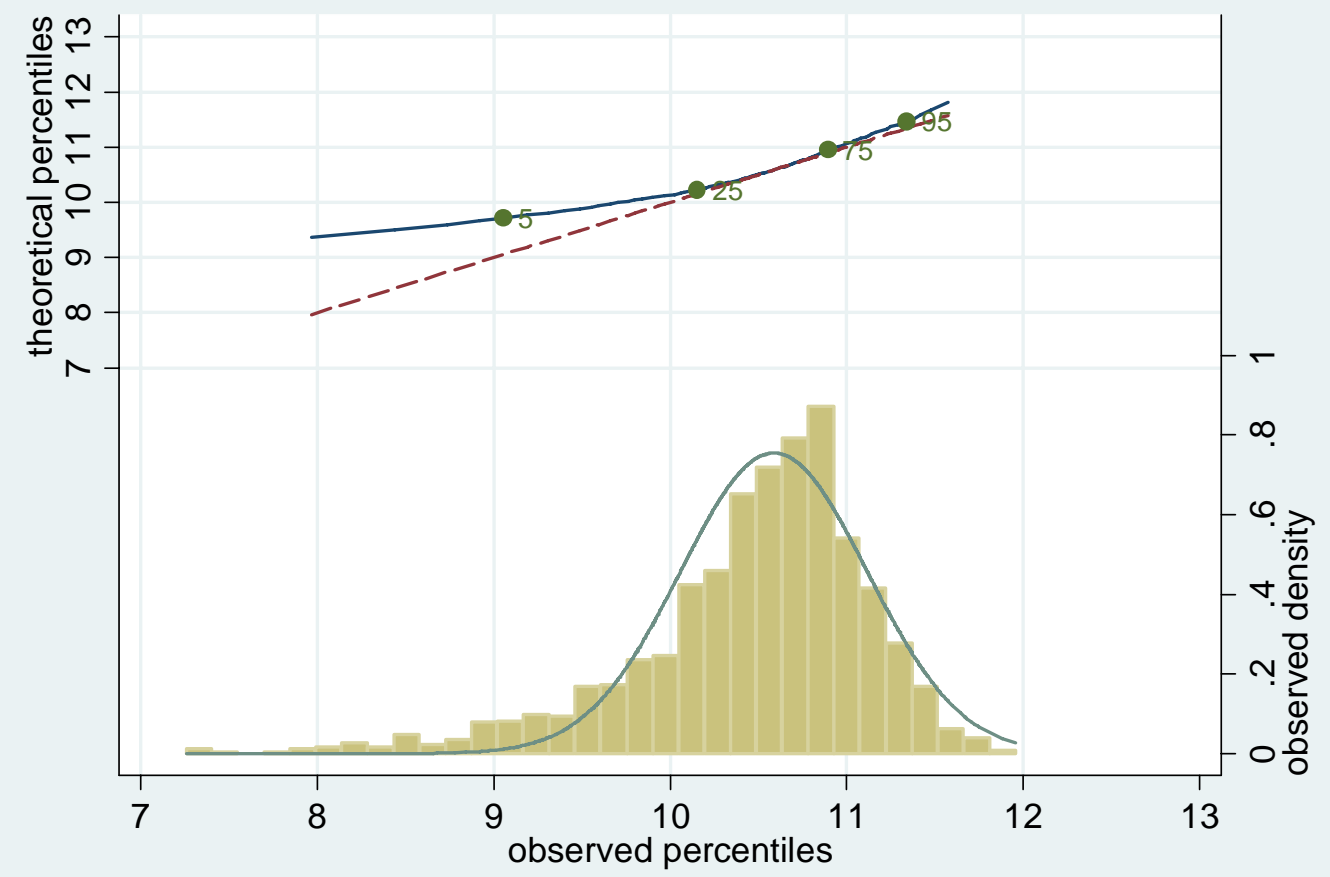

Standard Deviation of Logs: 0.5284 Skewness: -0.1725 Kurtosis: 0.2373

P-values: Kolmogorov-Smirnov: 0.0000 Skewness: 0.0000 Kurtosis: 0.0000 


\section{Age 51-55, expenditure}

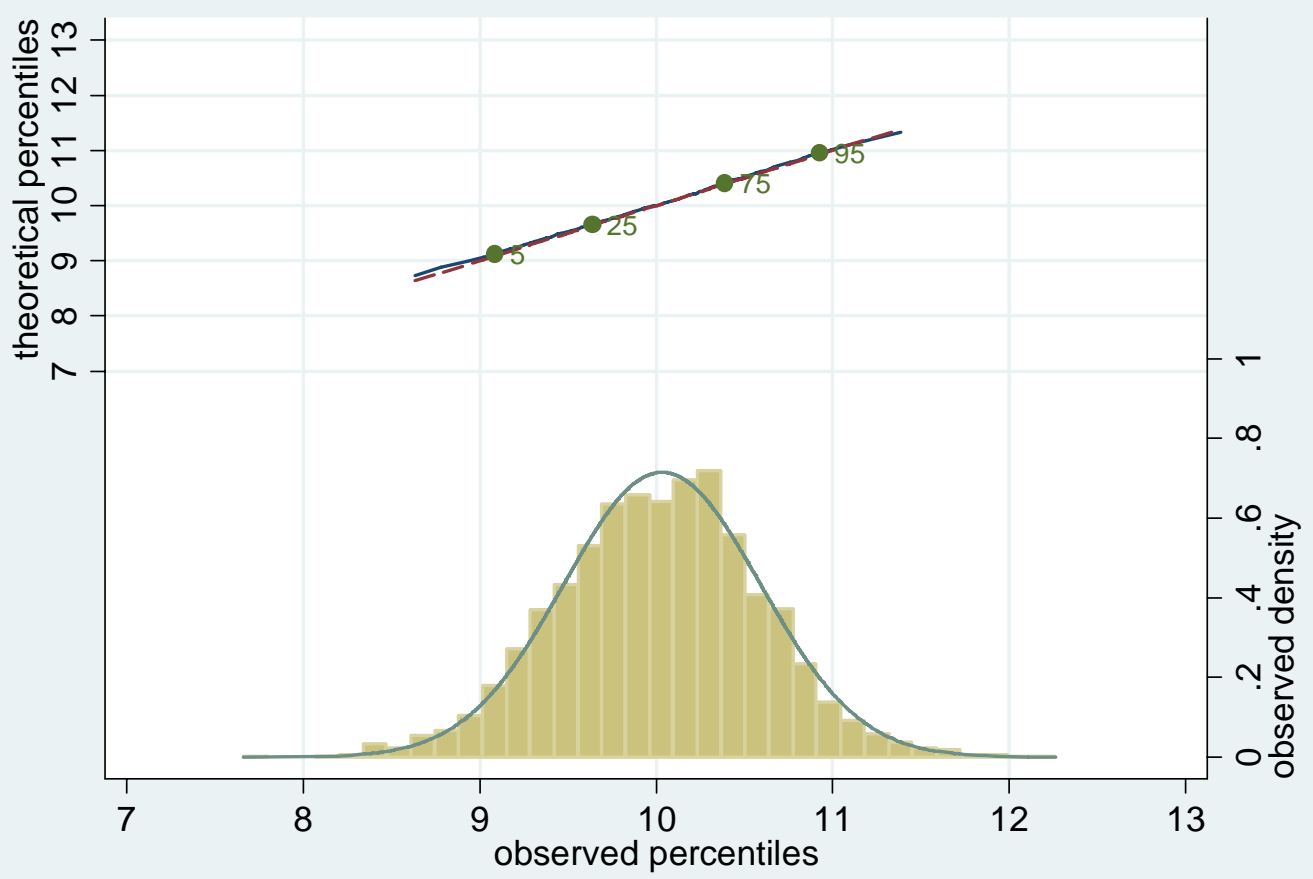

Standard Deviation of Logs: 0.5578 Skewness: -0.0491 Kurtosis: -0.0127

P-values: Kolmogorov-Smirnov: 0.2778 Skewness: 0.1190 Kurtosis: 0.7076

\section{Age 51-55, income}

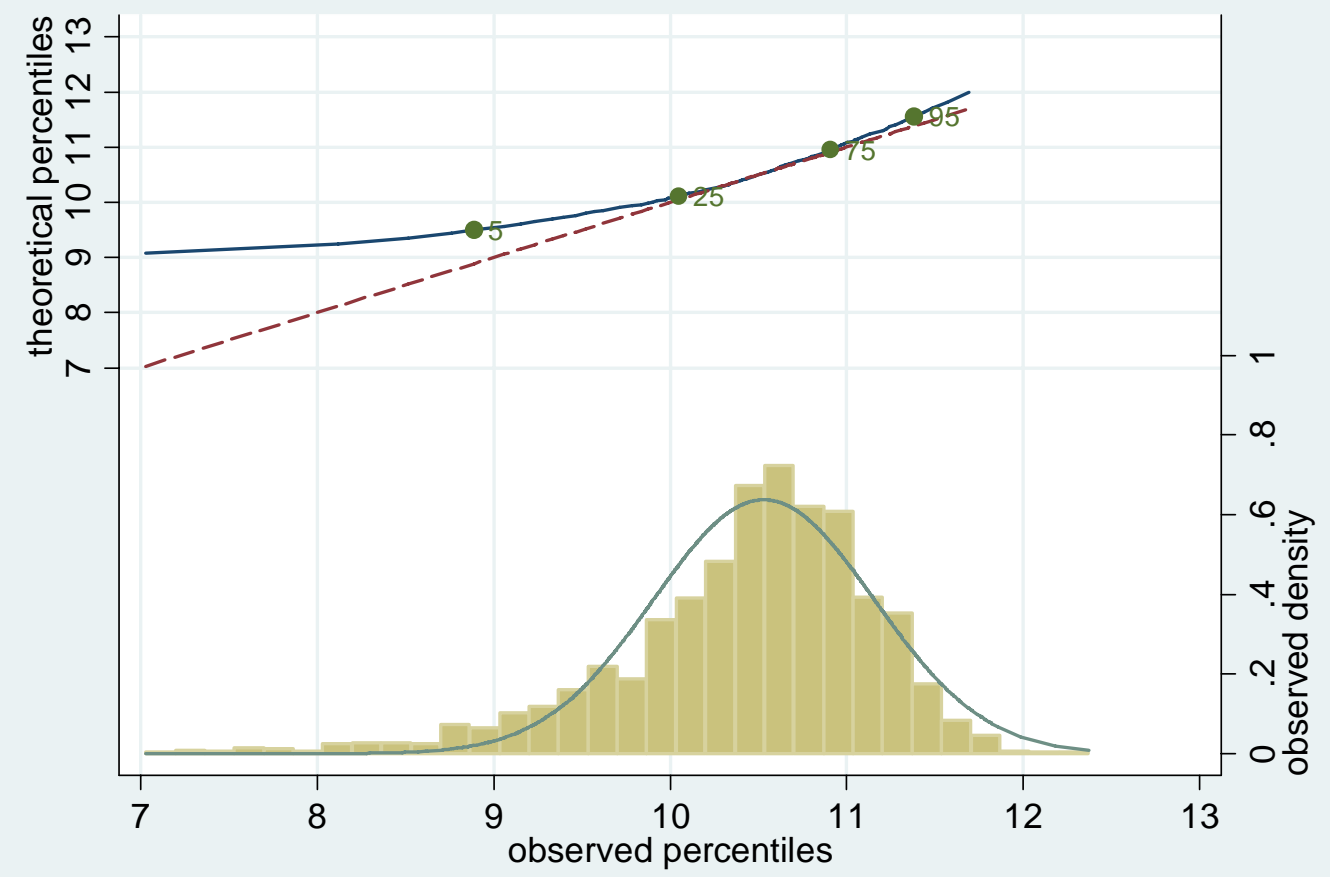

Standard Deviation of Logs: 0.6258 Skewness: -0.1210 Kurtosis: 0.1962

P-values: Kolmogorov-Smirnov: 0.0000 Skewness: 0.0010 Kurtosis: 0.0000 
Figure 7: The 1930-39 Birth Cohort from the CEX

\section{Age 51-55, expenditure}

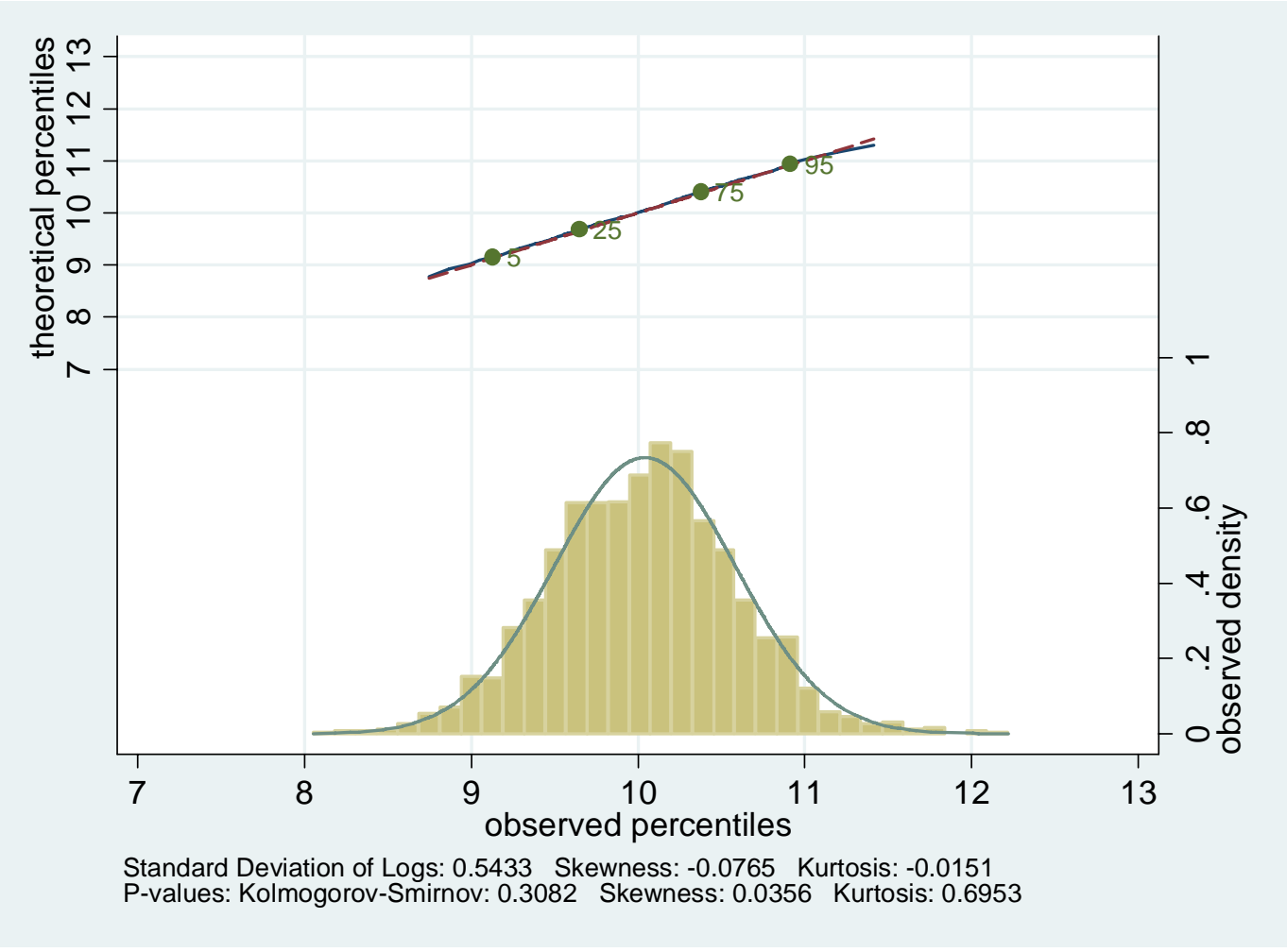

\section{Age 51-55, income}

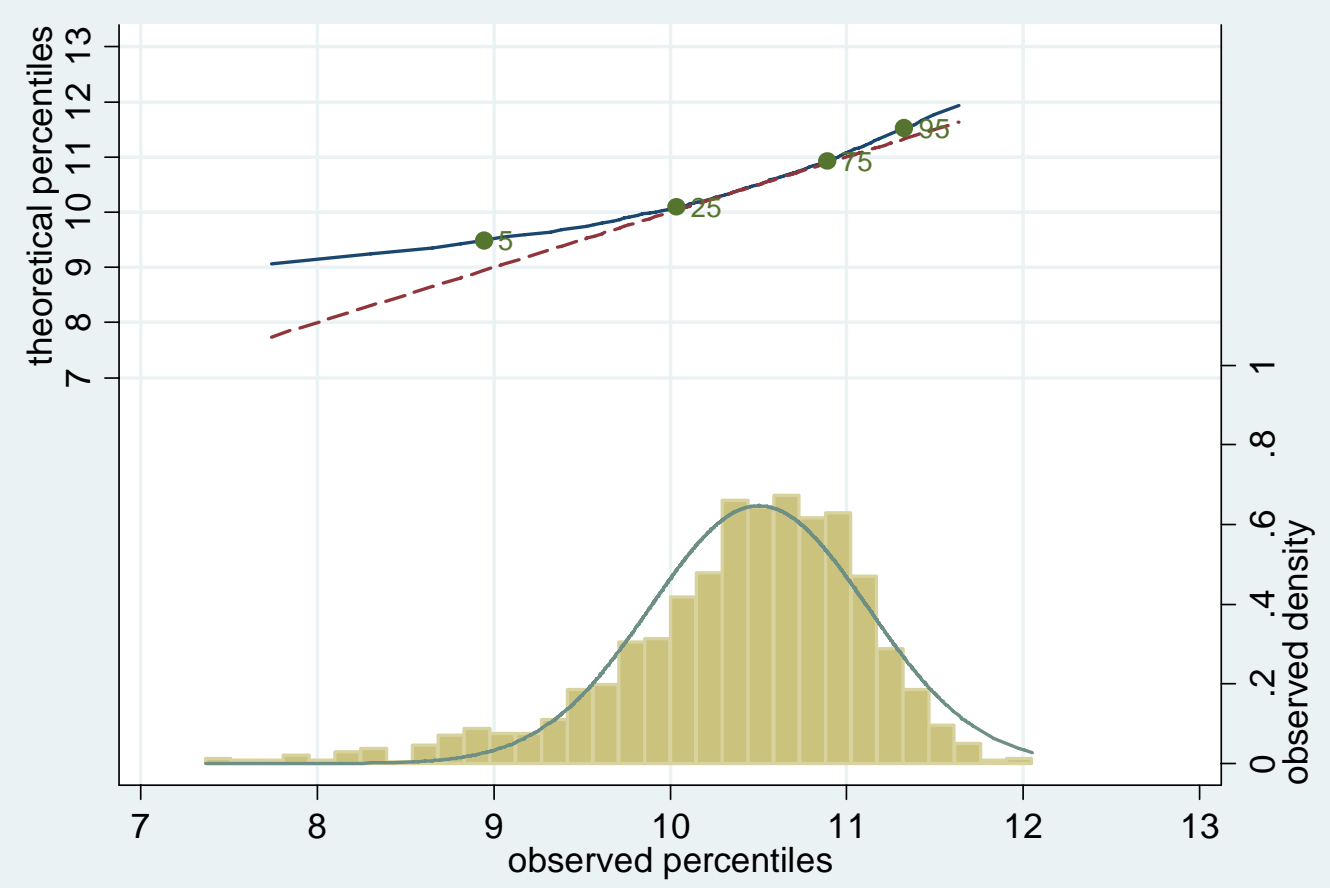

Standard Deviation of Logs: 0.6163 Skewness: -0.0978 Kurtosis: 0.0901

P-values: Kolmogorov-Smirnov: 0.0000 Skewness: 0.0151 Kurtosis: 0.0379 


\section{Age 56-60, expenditure}

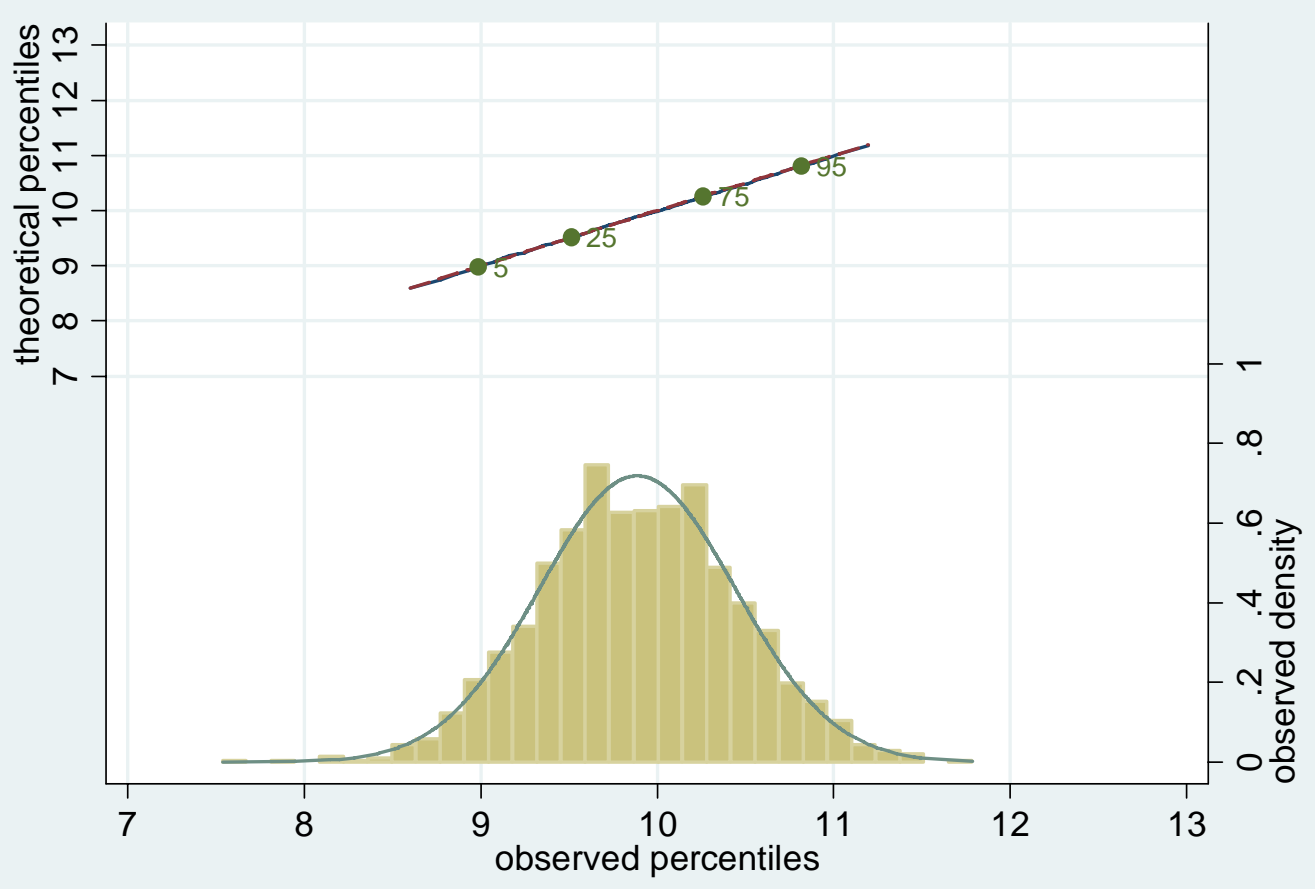

Standard Deviation of Logs: 0.5549 Skewness: 0.0063 Kurtosis: -0.0266

P-values: Kolmogorov-Smirnov: 0.7297 Skewness: 0.8879 Kurtosis: 0.5704

\section{Age 56-60, income}

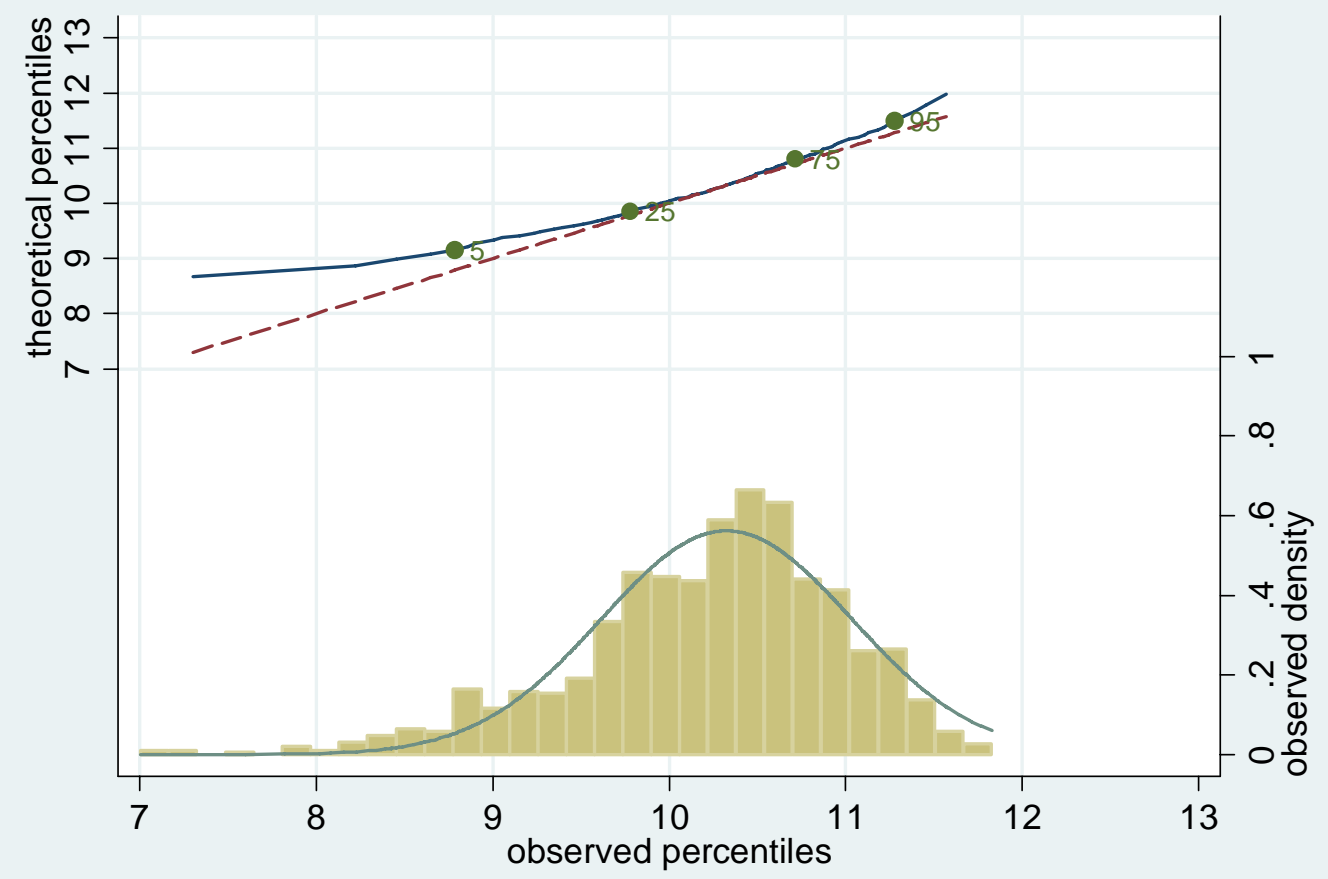

Standard Deviation of Logs: 0.7096 Skewness: -0.1677 Kurtosis: 0.0958

P-values: Kolmogorov-Smirnov: 0.0021 Skewness: 0.0008 Kurtosis: 0.0662 


\section{Age 61-65, expenditure}

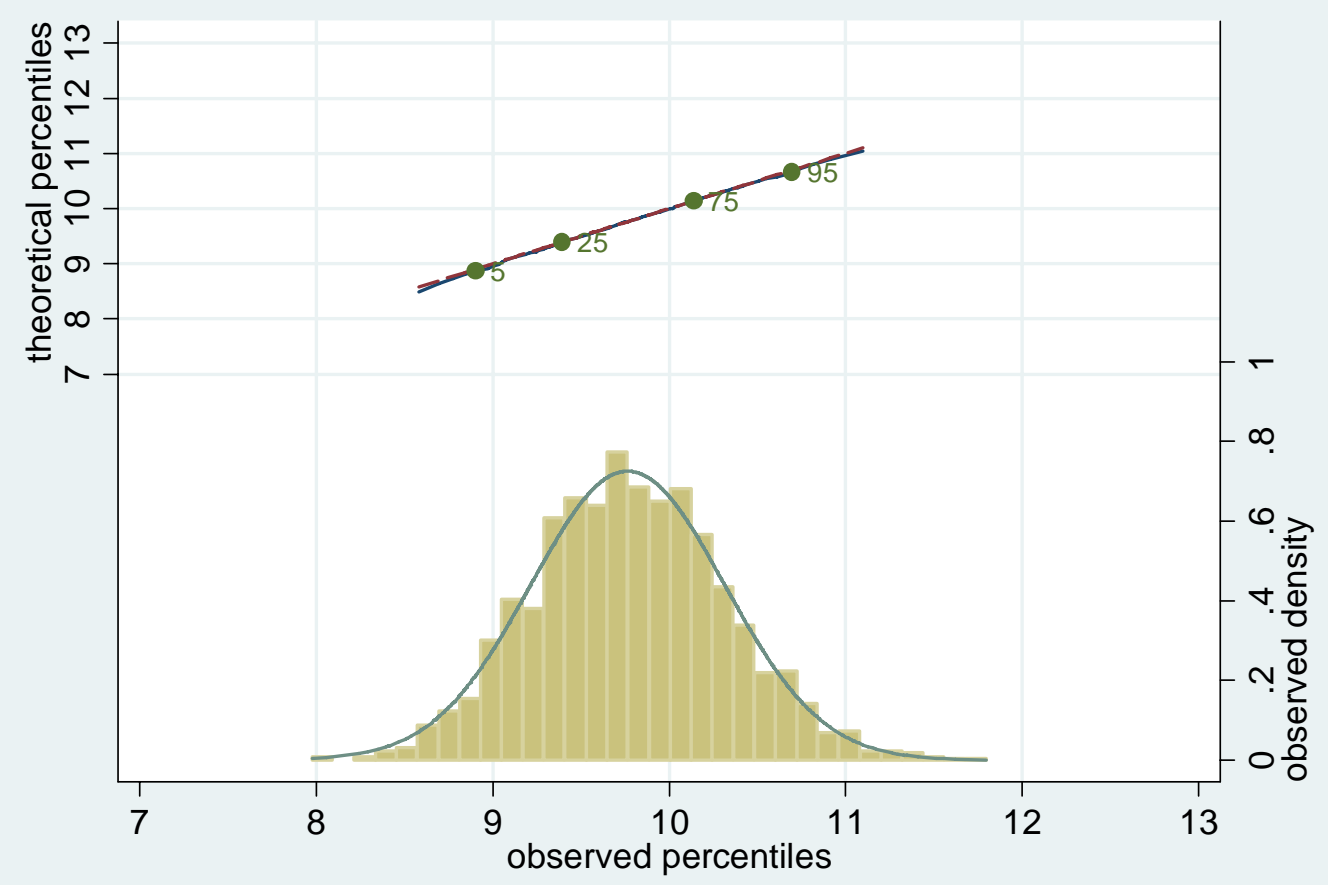

Standard Deviation of Logs: 0.5499 Skewness: 0.0042 Kurtosis: -0.0295

P-values: Kolmogorov-Smirnov: 0.5953 Skewness: 0.9141 Kurtosis: 0.4678

\section{Age 61-65, income}

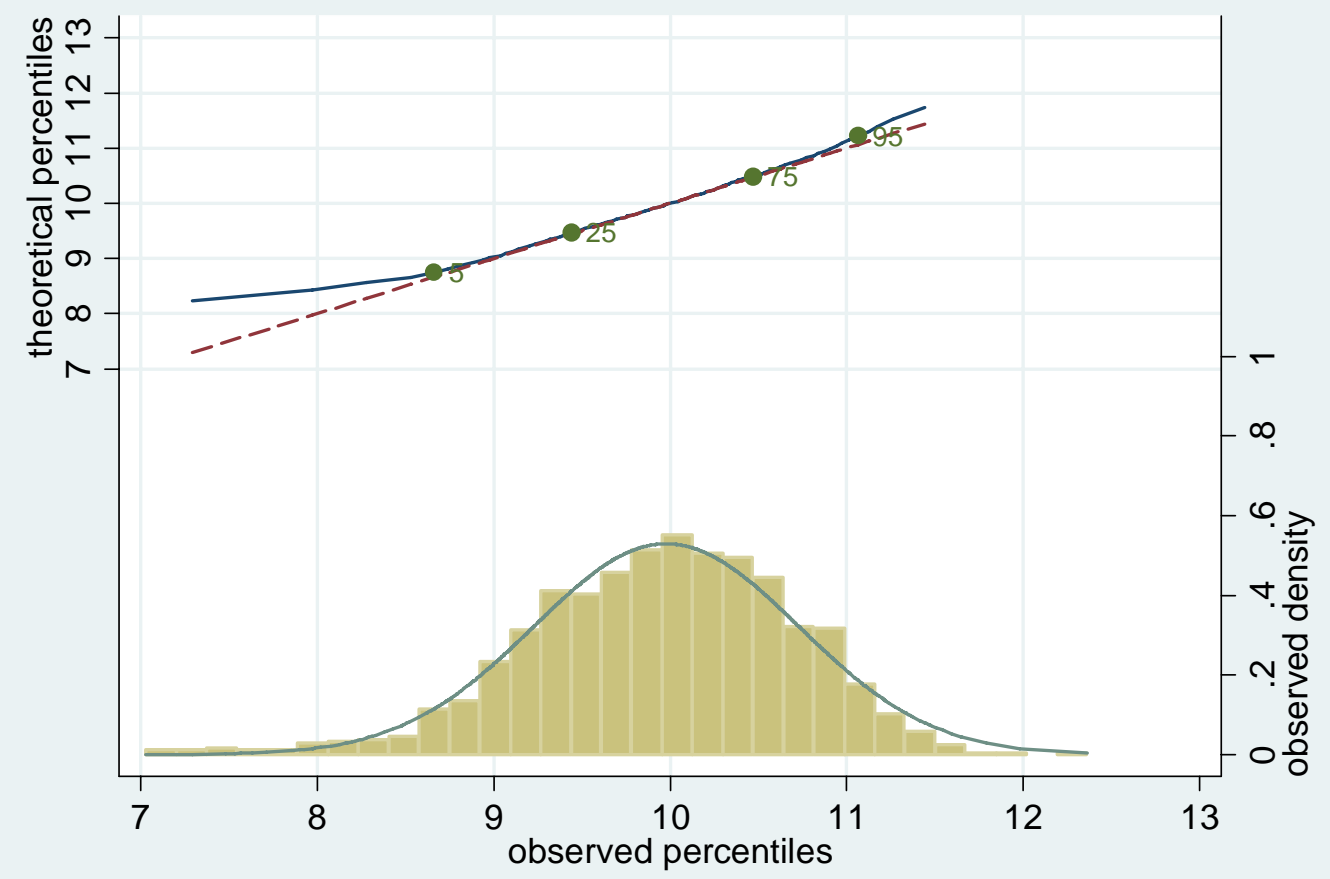

Standard Deviation of Logs: 0.7532 Skewness: -0.0455 Kurtosis: -0.0572

P-values: Kolmogorov-Smirnov: 0.1729 Skewness: 0.2952 Kurtosis: 0.2254 
Figure 8: Young Households in the 1960-69 Birth Cohort from the CEX

(a) Age 21-25, expenditure

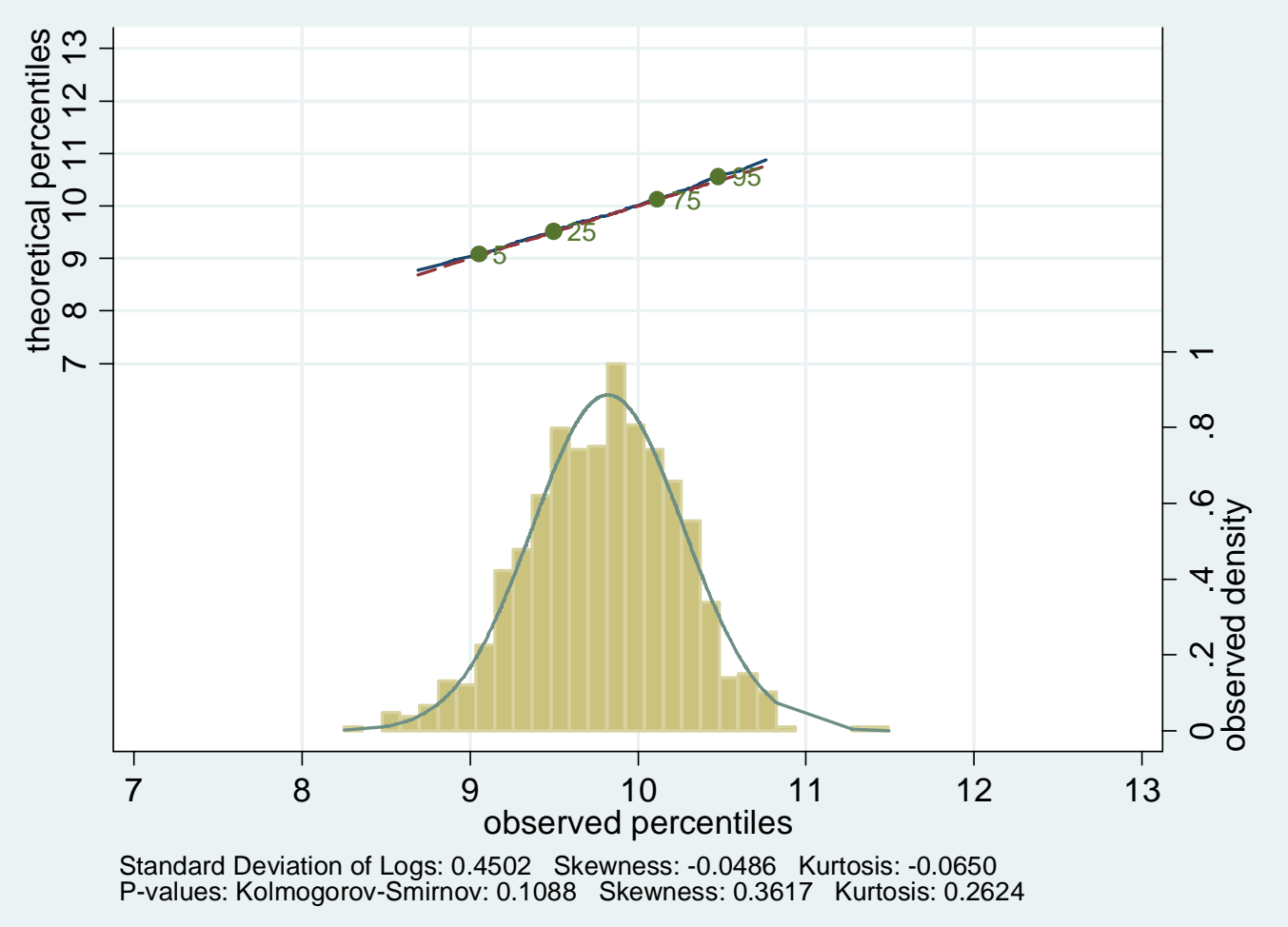

(b) Age 21-25, income

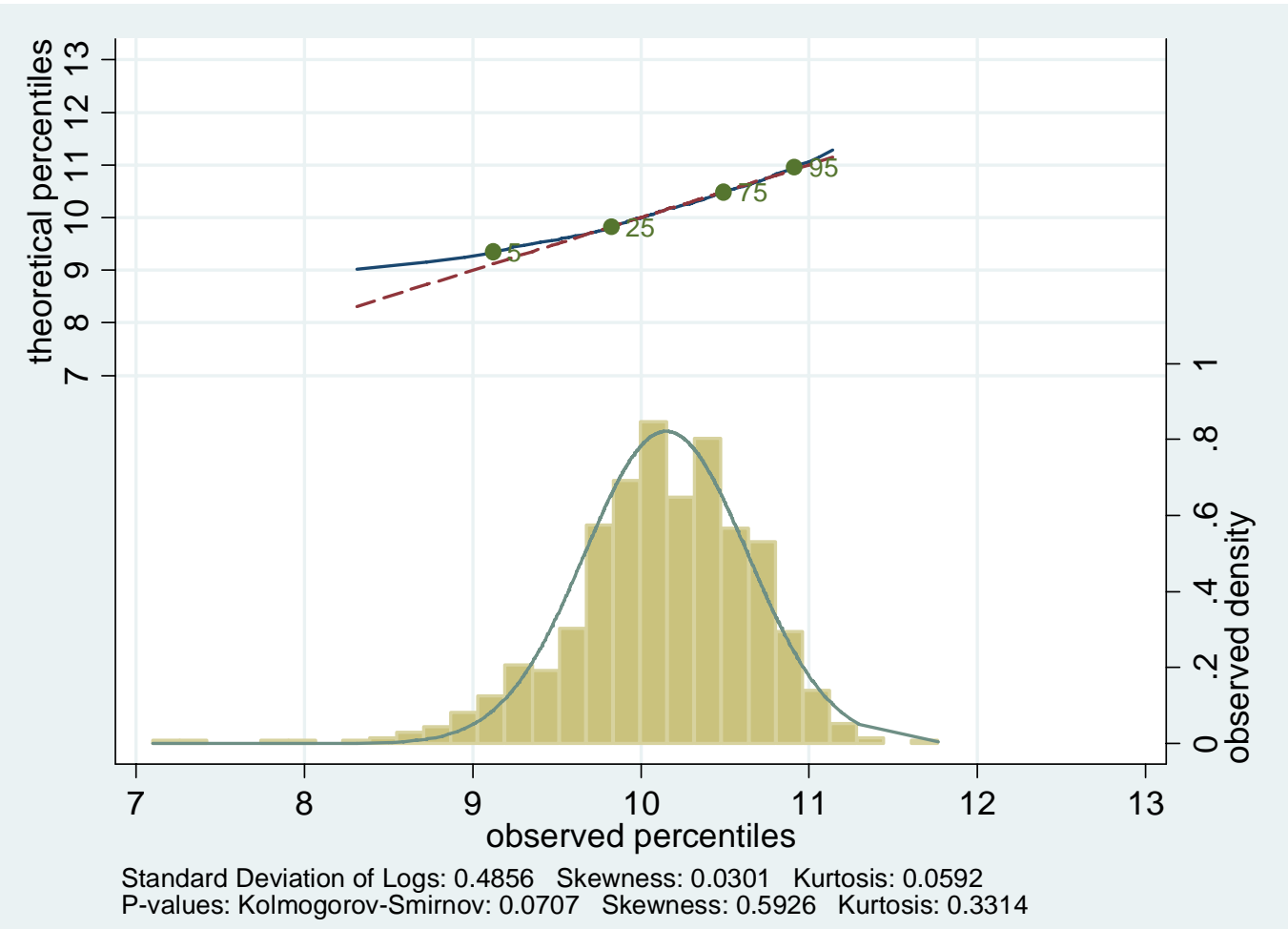


Figure 9: Family Income in the PSID

(a) The 1940-1949 Birth Cohort

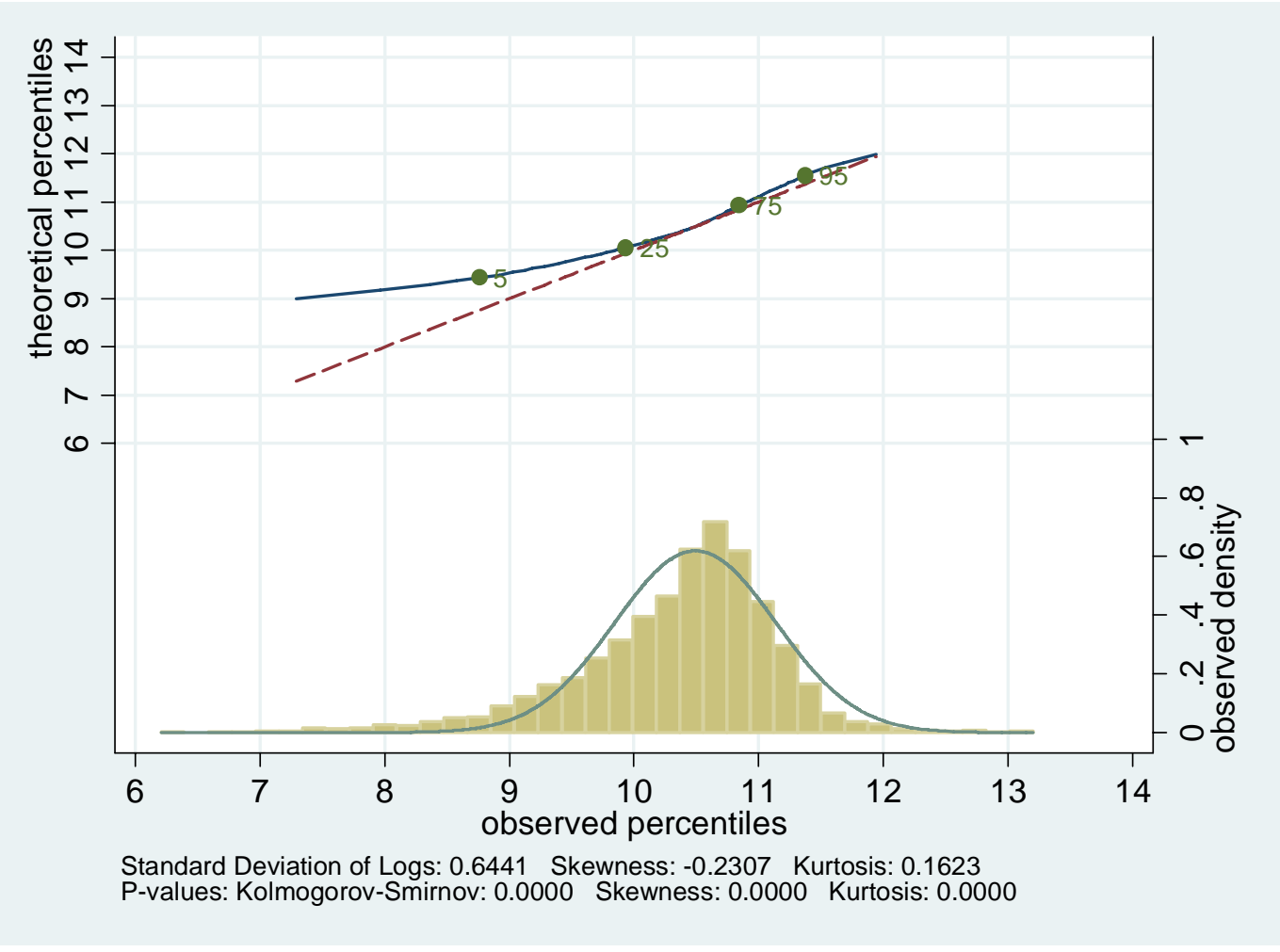

\section{(b) The 1930-1939 Birth Cohort}

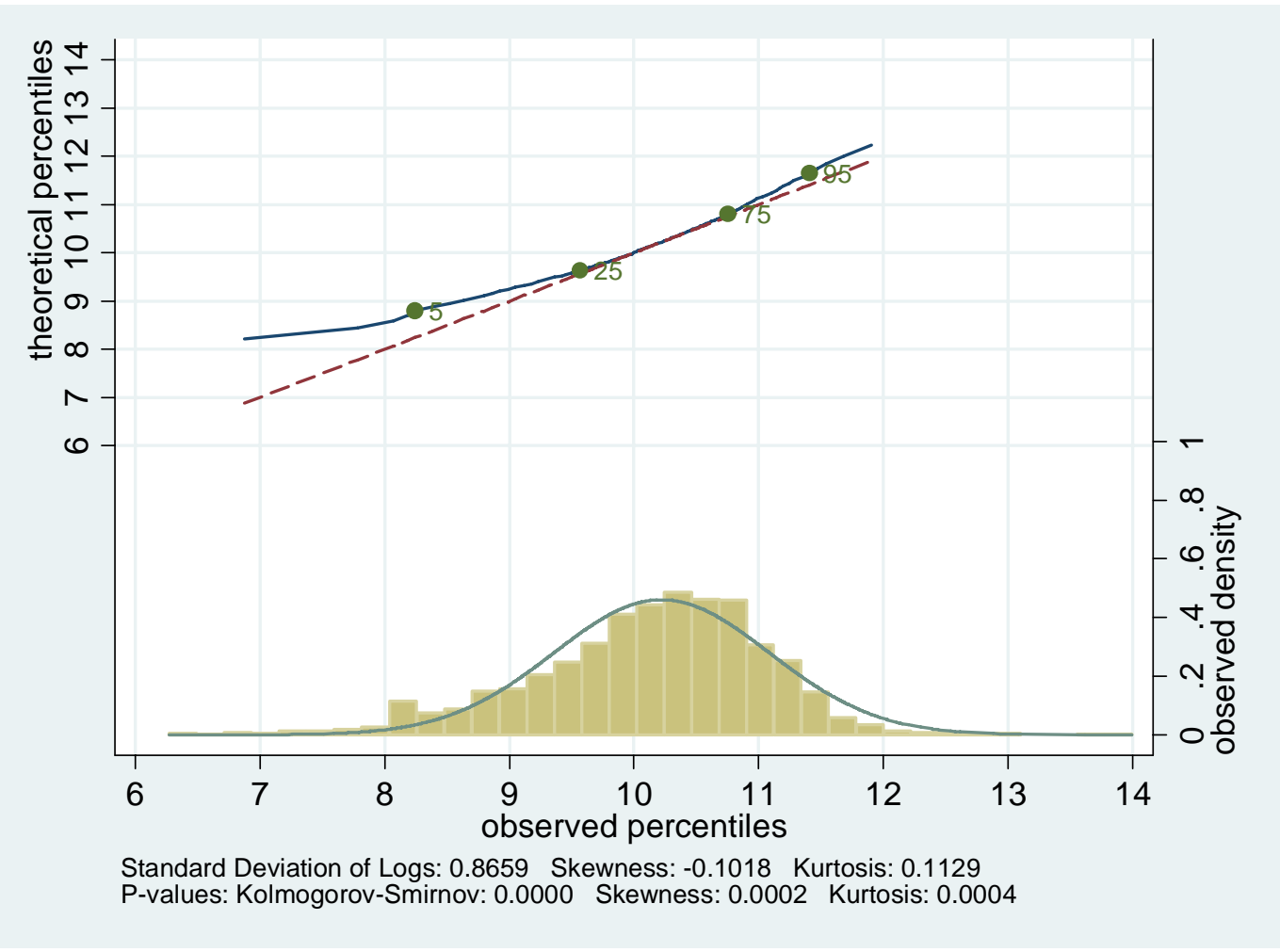


Figure 10: Family Expenditure in the FES

(a) COHORT 1950-59, AGE 41-45

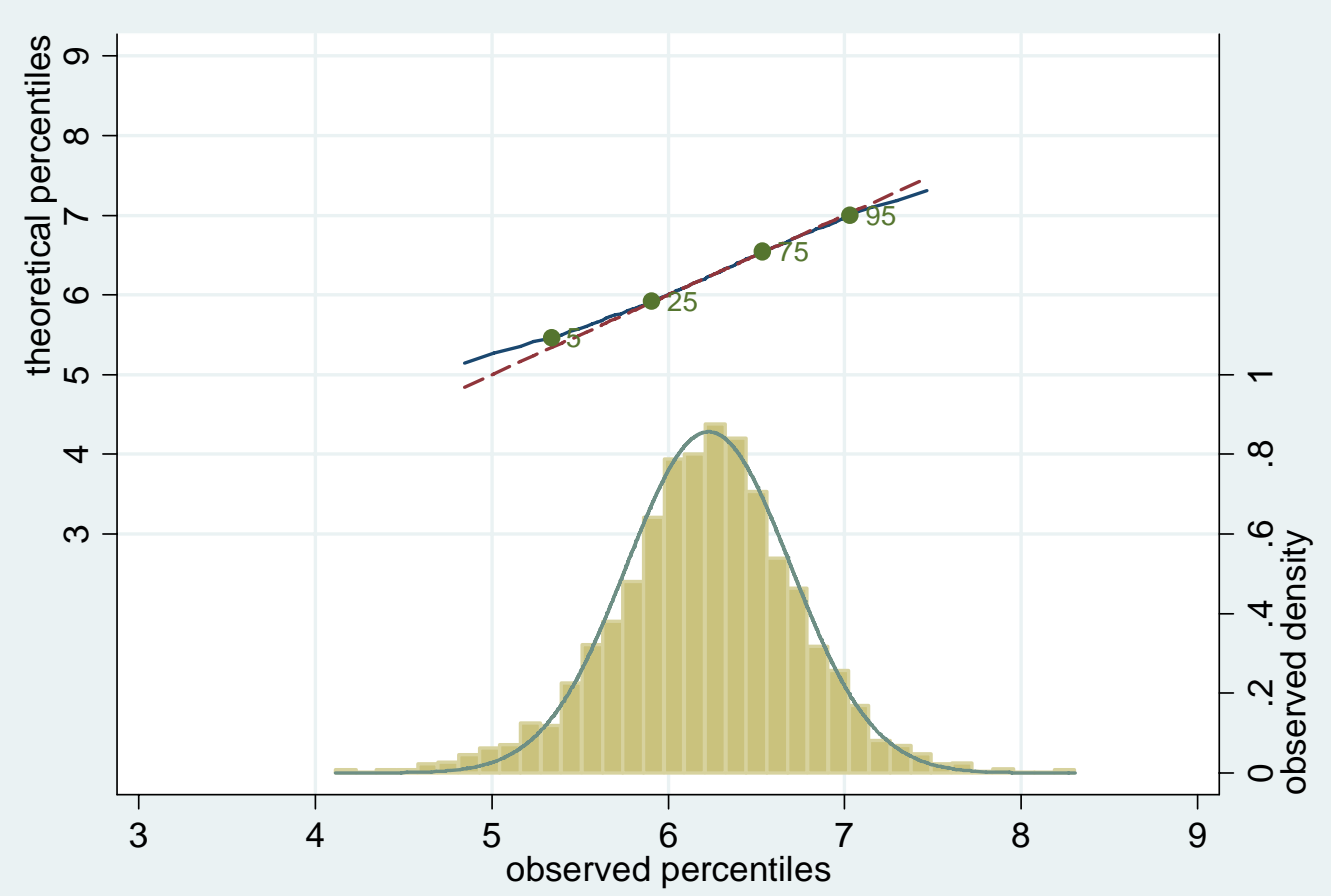

Standard Deviation of Logs: 0.4657 Skewness: -0.0198 Kurtosis: 0.1142

P-values: Kolmogorov-Smirnov: 0.0082 Skewness: 0.4438 Kurtosis: 0.0002

(b) COHORT 1940-49, AGE 41-45

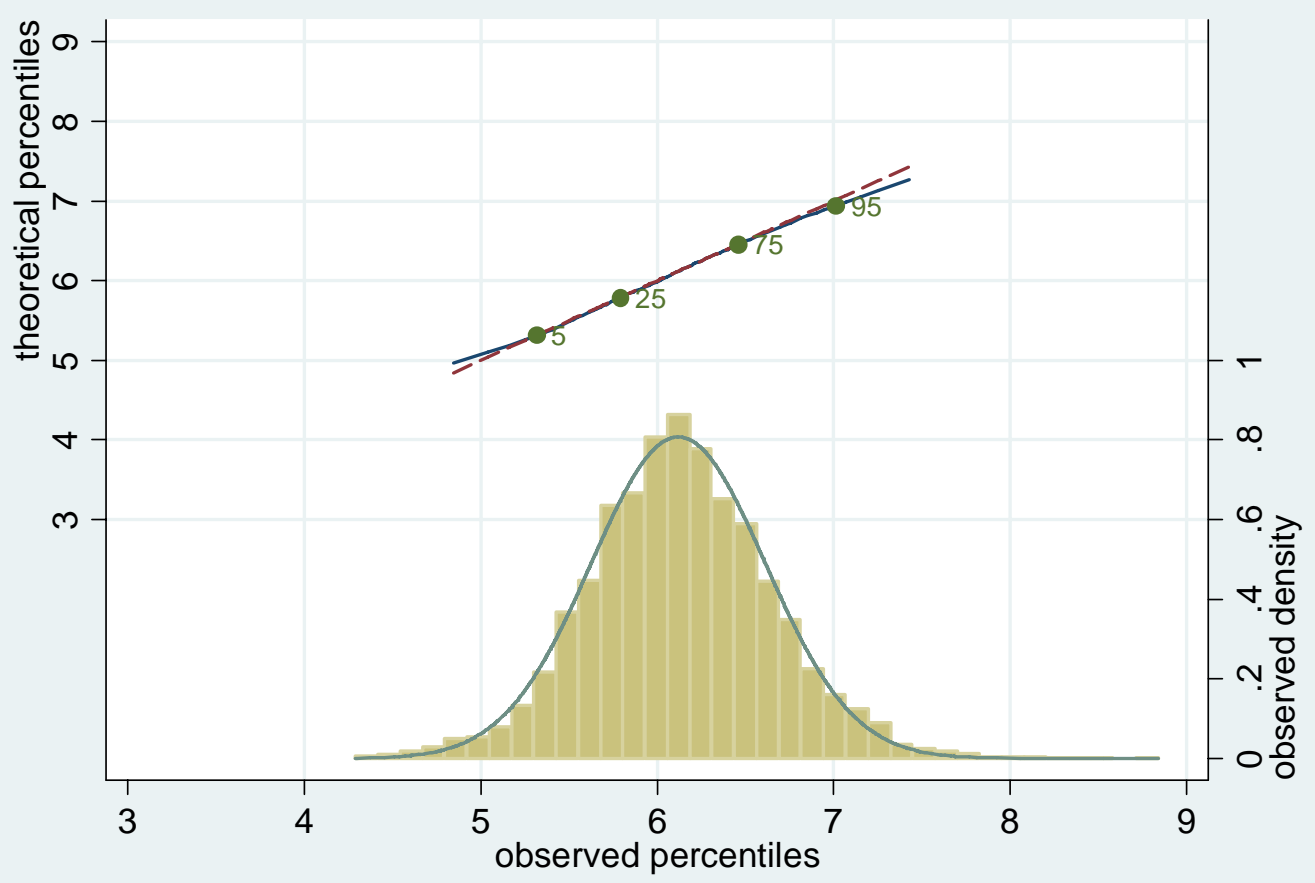

Standard Deviation of Logs: 0.4941 Skewness: 0.0192 Kurtosis: 0.0474

P-values: Kolmogorov-Smirnov: 0.1684 Skewness: 0.4113 Kurtosis: 0.0645 
(c) COHORT 1940-49, AGE 51-55

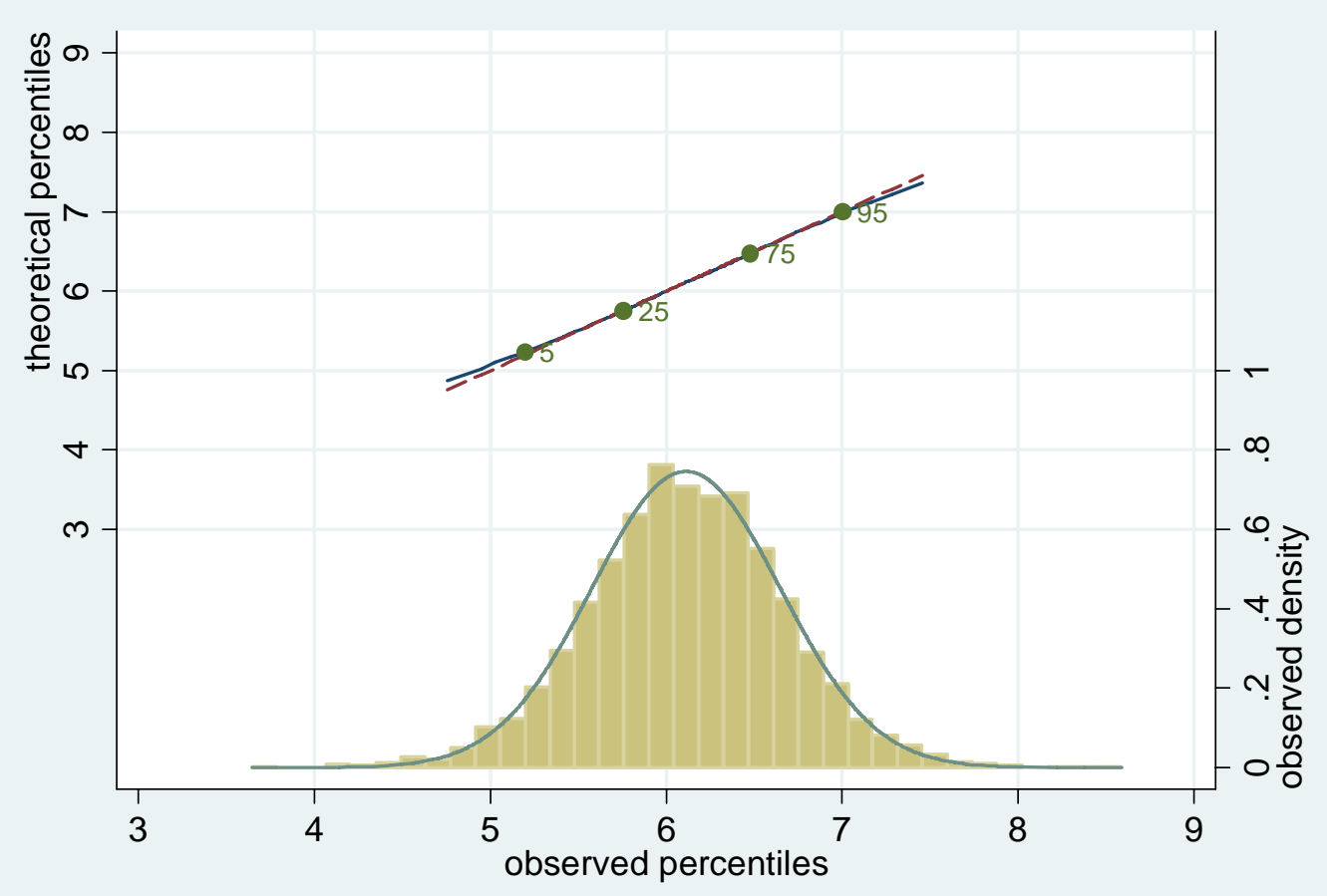

Standard Deviation of Logs: 0.5348 Skewness: 0.0132 Kurtosis: -0.0030

P-values: Kolmogorov-Smirnov: 0.6692 Skewness: 0.6280 Kurtosis: 0.9192

(d) COHORT 1930-39, AGE 51-55

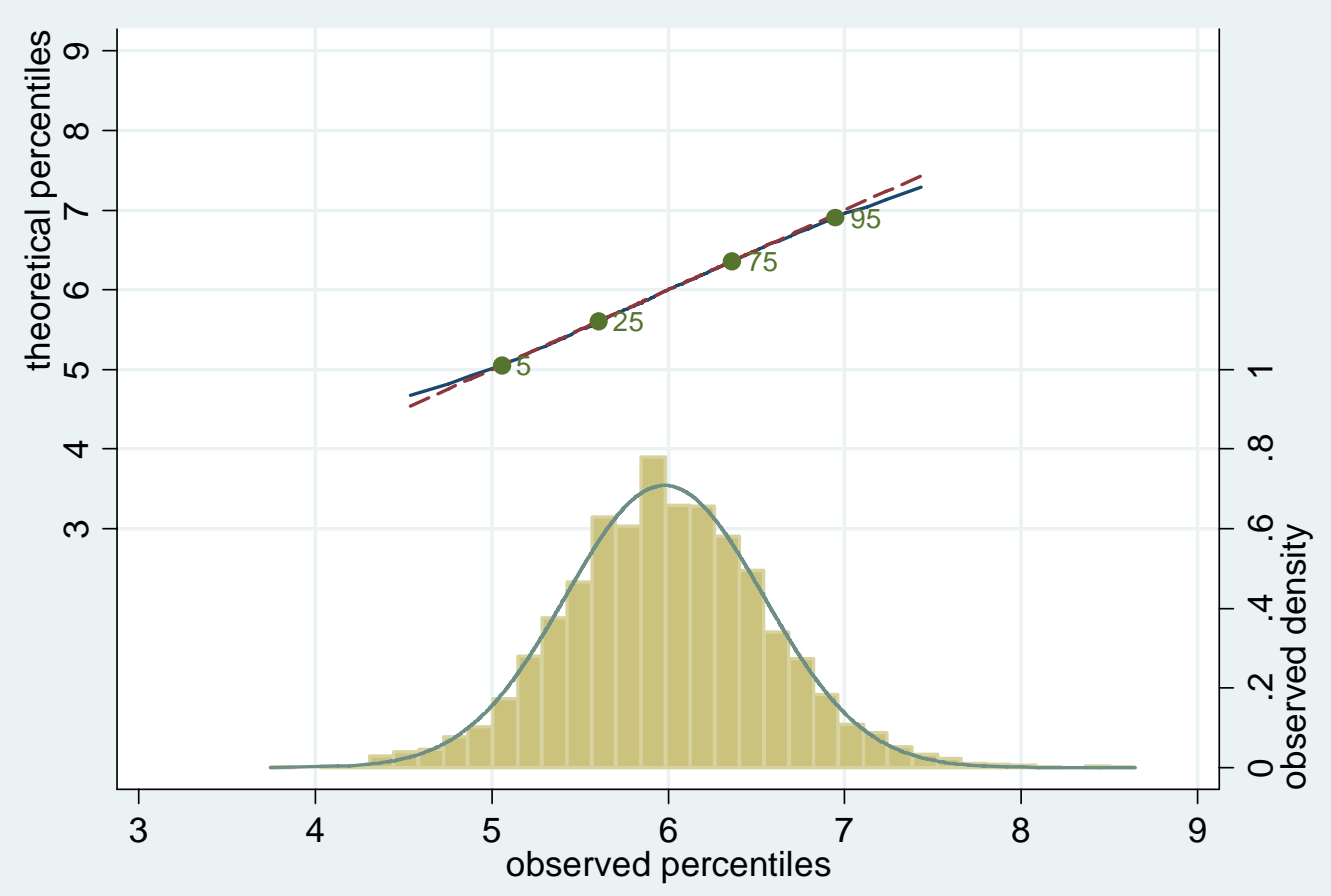

Standard Deviation of Logs: 0.5629 Skewness: 0.0102 Kurtosis: 0.0012

P-values: Kolmogorov-Smirnov: 0.8702 Skewness: 0.7089 Kurtosis: 0.9681 
Figure 11: Expenditure Distributions using a $\sqrt{n}$ equivalence scale

(a) COHORT 1950-59, AGE 41-45 - CEX EXP DATA

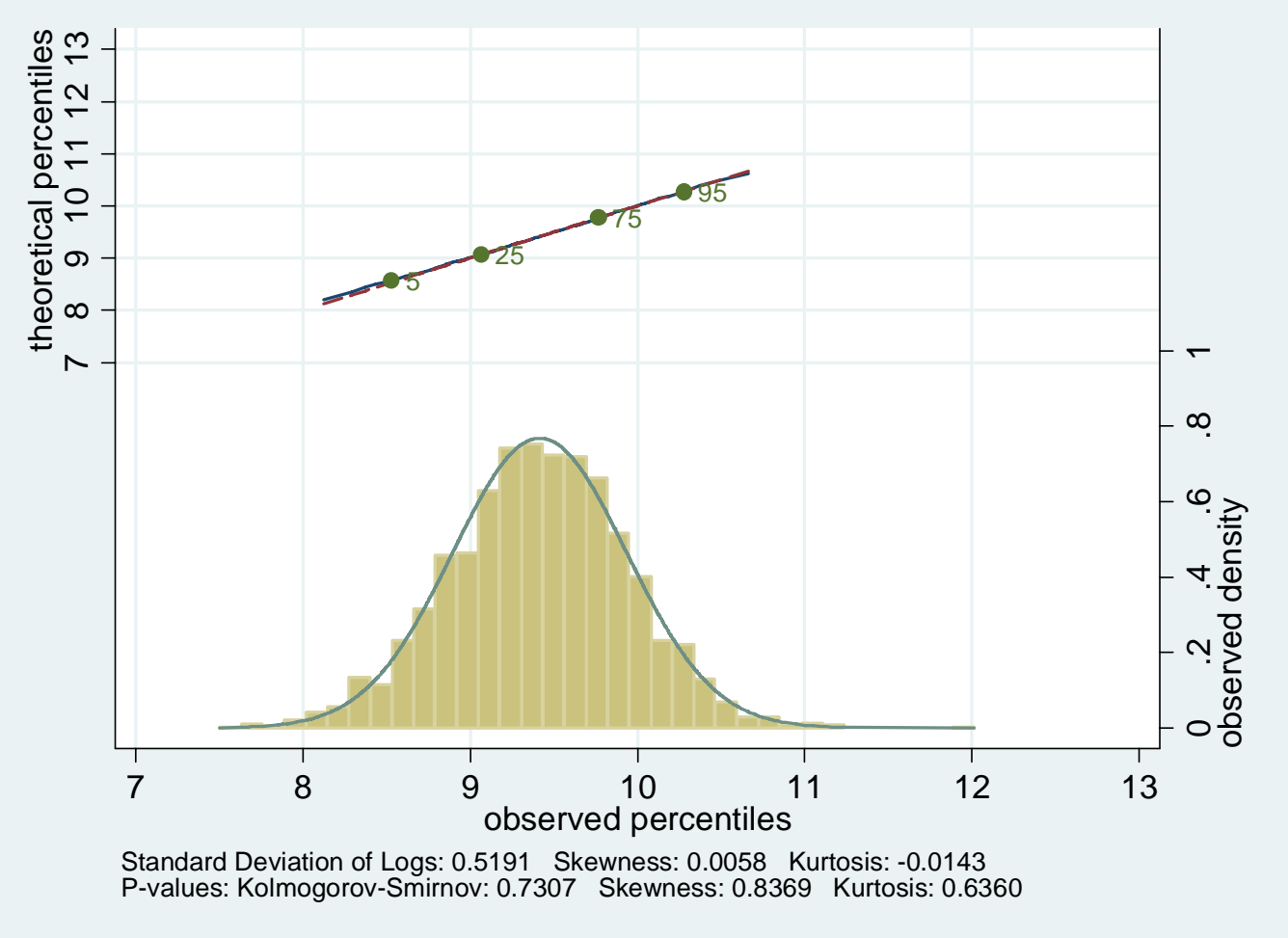

(b) COHORT 1940-49, AGE 41-45 - CEX EXP DATA

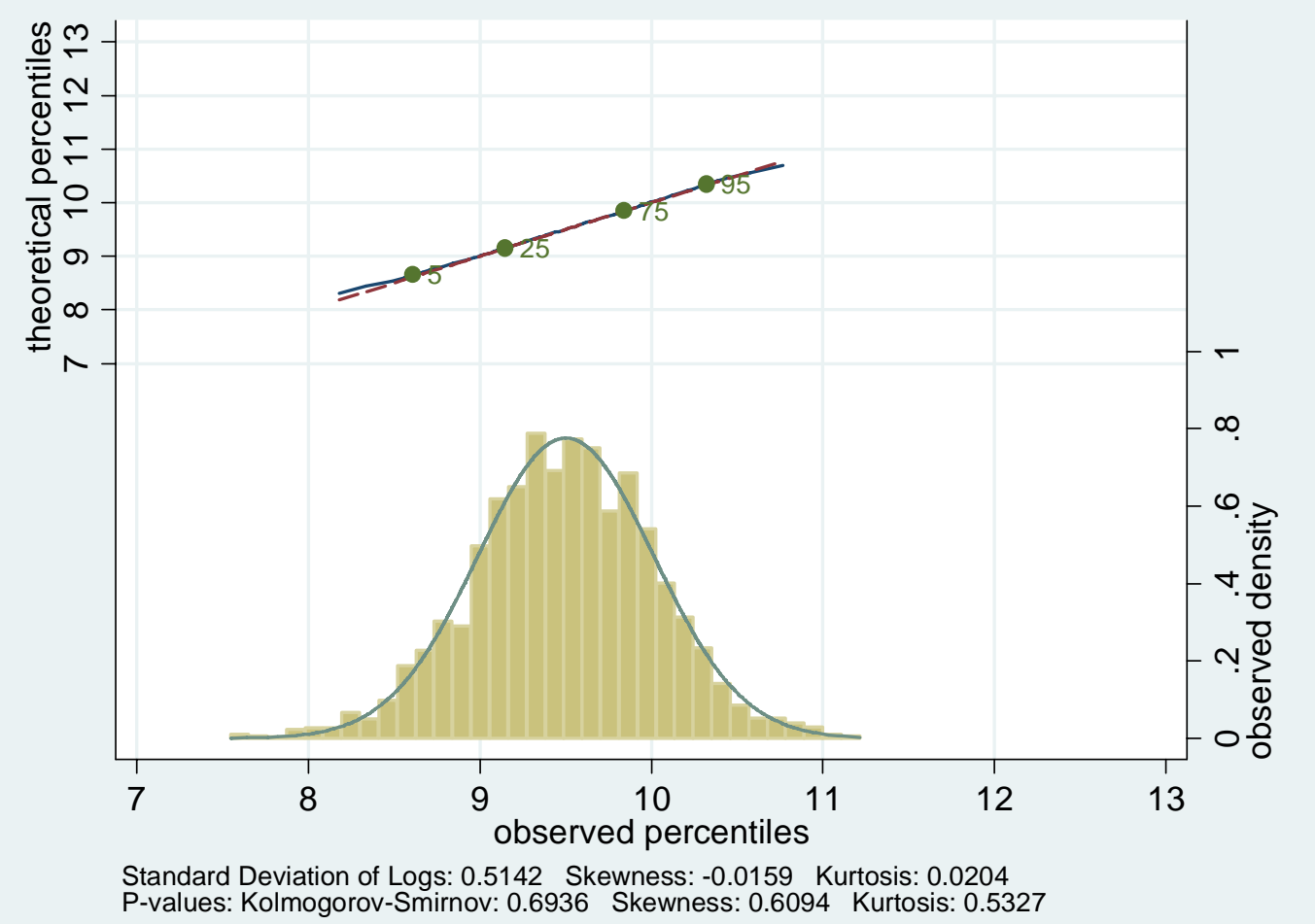


(c) COHORT 1940-49, AGE 51-55 - CEX EXP DATA

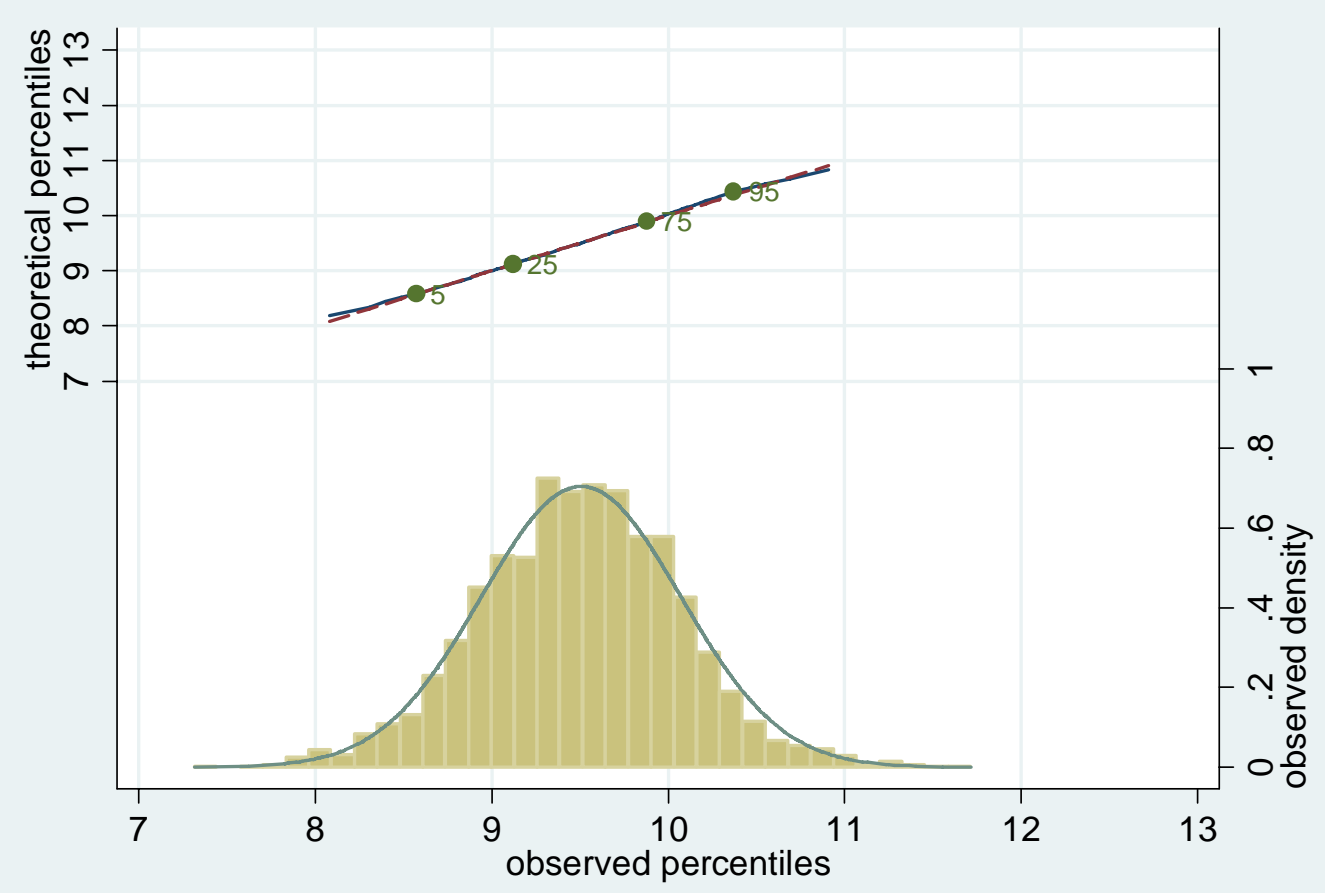

Standard Deviation of Logs: 0.5660 Skewness: -0.0204 Kurtosis: -0.0463

P-values: Kolmogorov-Smirnov: 0.3208 Skewness: 0.5167 Kurtosis: 0.1726

(d) COHORT 1930-39, AGE 51-55 - CEX EXP DATA

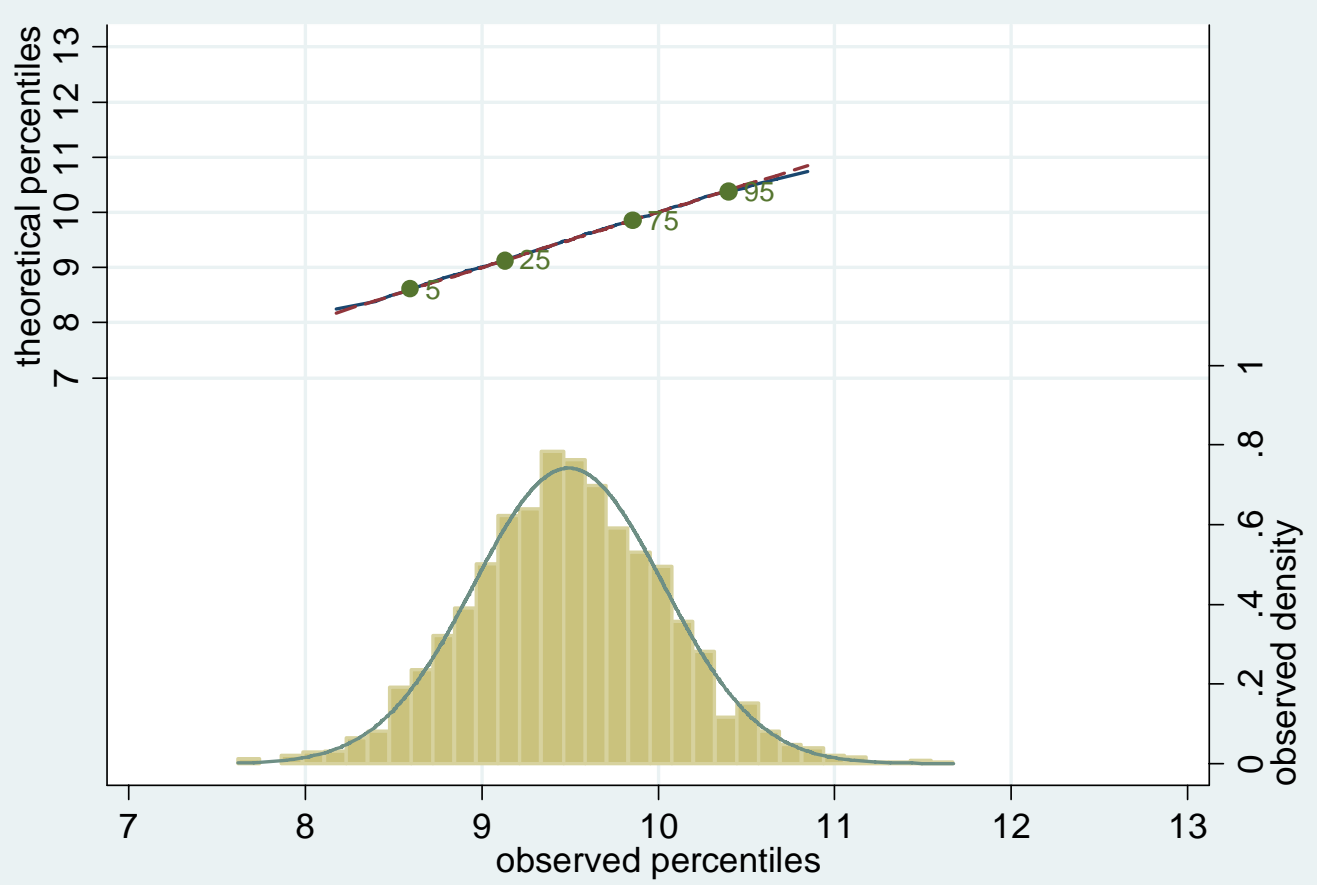

Standard Deviation of Logs: 0.5376 Skewness: 0.0050 Kurtosis: 0.0565

P-values: Kolmogorov-Smirnov: 0.9500 Skewness: 0.8915 Kurtosis: 0.1515 